\title{
Normal Positive Linear Mappings of Norm 1 from a von Neumann Algebra into Its Commutant and Its Application
}

By

\author{
Huzihiro Araki*
}

\begin{abstract}
Let $M$ and $N$ be von Neumann algebras such that $N \subset M^{\prime}$. Let $Z=N \cap M$ and $\rho$ be any normal positive linear functional of $(M \cup N)^{\prime \prime}$. There exists a unique mapping $F_{\rho}^{N M}$ from $M$ into $N$ satisfying

$$
(1 / 2) \rho\left(\mathrm{F}_{\rho}^{N M}\left(Q_{1}\right) Q_{2}+Q_{2} F_{\rho}^{N M}\left(Q_{1}\right)\right)=\rho\left(Q_{1} Q_{2}\right)
$$

for all $Q_{1} \in M, Q_{2} \in N$ and $s\left(F_{\rho}^{N M}\left(Q_{1}\right)\right) \leqq s^{N}(\rho)$, where $s$ denotes the support and $s^{N}$ denotes the support in $N$. The mapping $F_{\rho}^{N M}$ is $Z$-linear, positive and transposed- $n$-positive, of norm 1 and continuous on the unit ball weakly and strongly.

As an application, a generalization of a clustering theorem for an asymptotically abelian case is given.
\end{abstract}

\section{$\S 1$. Preliminaries}

We consider two von Neumann algebras $M$ and $N$ such that $N \subset M^{\prime}$ and a normal positive linear functional $\rho$ of $(M \cup N)^{\prime \prime} . \quad H_{\rho}, \pi_{\rho}$, and $\Omega_{\rho}$ denote a Hilbert space, a representation of $(M \cup N)^{\prime \prime}$ and a cyclic vector canonically associated with $\rho$ through $\rho=\omega_{\Omega_{\rho}}$ where $\omega_{\Omega}$ denotes the expectation functional by the vector $\Omega$ (called a vector state if $\omega_{\Omega}(1)=1$ ).

$s(A)$ for an operator $A$ on a Hilbert space denotes the support of $A$, namely the smallest projection $E$ satisfying $E A=A E=A . \quad s(A)$ is in the von Neumann algebra generated by $A$ and $A^{*}$ and hence the notation $s(A)$ is also used for an element of von Neumann algebra. $s^{N}(\rho)$ denotes

Received June 22, 1972.

* On leave from Research Institute for Mathematical Sciences, Kyoto University, Kyoto, Japan. 
the support of $\rho$ relative to $N$, namely the smallest projection $E$ in $N$ such that $\rho(E)=\rho(1) . \quad s^{N}(\Omega)$ denotes $s^{N}\left(\omega_{\Omega}\right)$.

Our tool is the following version of the Radon-Nikodym theorem by Sakai [6].

Lemma $\mathbb{1}$. Let $\mu$ and $\nu$ be normal positive linear functionals of $a$ von Neumann algebra $N$ such that $\mu \geqq \nu$. There exists a unique $h_{0} \in N$ satisfying

(1) $\nu(Q)=(1 / 2) \mu\left(h_{0} Q+Q h_{0}\right), \quad Q \in N$,

(2) $s\left(h_{0}\right) \leqq s^{N}(\ell)$

(3) $0 \leqq h_{0} \leqq 1$

Proof. The existence of $h_{0}$ satisfying (1) and (3) is in [6]. Since $0 \leqq \nu\left(1-s^{N}(\mu)\right) \leqq \mu\left(1-s^{N}(\mu)\right)=0$, we have $s^{N}(\nu) \leqq s^{N}(\mu)$. Setting $Q=$ $s^{N}(\mu) h_{0}\left(1-s^{N}(\mu)\right)$, we obtain from (1)

$$
0=\nu(Q)=(1 / 2) \mu\left(Q h_{0}\right)=(1 / 2) \mu\left(Q Q^{*}\right) .
$$

Since $s^{N}(\mu) Q Q^{*} s^{N}(\mu)=Q Q^{*}$, we obtain $Q Q^{*}=0$, i.e. $Q=Q^{*}=0$. Hence

$$
h_{0}=h_{0}^{\prime}+h_{0}^{\prime \prime}
$$

where $h_{0}^{\prime}=s^{N}(\mu) h_{0} s^{N}(\mu)$ and $h_{0}^{\prime \prime}=\left(1-s^{N}(\mu)\right) h_{0}\left(1-s^{N}(\mu)\right)$. Since

$$
\mu\left(h_{0} Q+Q h_{0}\right)=\mu\left(h_{0}^{\prime} Q+Q h_{0}^{\prime}\right)
$$

$h_{0}^{\prime} \in N$ satisfies (1), (2) and (3).

The uniqueness holds in the following slightly more general form.

Q.E.D.

Lemma 2. Let $\mu$ and $\nu$ be normal linear functionals of $N$ and $\mu$ be positive. An operator $h_{0} \in N$ satisfying (1) and (2) of Lemma 1 is unique, if it exists.

Proof. Suppose $h_{0}$ and $h_{0}^{\prime}$ satisfy (1) and (2). Then $h=h_{0}-h_{0}^{\prime}$ satisfy $\mu(h Q+Q h)=0$ for all $Q \in N$. Substituting $Q=h^{*}$, we have 


$$
0 \leqq \mu\left(h^{*} h\right) \leqq \mu\left(h h^{*}+h^{*} h\right)=0
$$

and hence $s^{N}(\mu) h^{*} h s^{N}(\mu)=0$. Since $s(h) \leqq s^{N}(\mu)$, we have $h^{*} h=0$ and hence $h_{0}-h_{0}^{\prime}=h=0$.

Q.E.D.

We use Lemma 1 in the following complex form.

Lemma 3. Let $\mu$ and $\nu$ be normal linear functionals of $N$,

$$
\nu=\nu_{1}-\nu_{2}+i\left(\nu_{3}-\nu_{4}\right)
$$

$\mu, \nu_{1}, \nu_{2}, \nu_{3}$, and $\nu_{4}$ be positive and $\nu_{k} \leqq \lambda \mu, k=1,2,3,4, \lambda>0$. There exists a unique $h_{0} \in N$ satisfying the conditions (1) and (2) of Lemma 1.

Proof. Immediate from Lemmas 1 and 2.

Q.E.D.

A linear mapping $F$ from a von Neumann algebra $M$ into $N$ is called $n$-positive if the mapping $F \otimes 1$ from $M \otimes \mathscr{B}\left(C^{n}\right)$ to $N \otimes \mathscr{B}\left(C^{n}\right)$ is positive, where $C^{n}$ is an $n$-dimensional Hilbert space, $\mathscr{B}\left(C^{n}\right)$ is the set of all linear operators on $C^{n}$ and $(F \otimes 1)\left(Q \otimes Q^{\prime}\right)=F(Q) \otimes Q^{\prime}$ for $Q \in M, Q^{\prime} \in$ $\mathscr{B}\left(C^{n}\right)$. If $F$ is $n$-positive for all positive integers $n, F$ is called completely positive.

$F$ is called transposed- $n$-positive if $F \otimes t$ from $M \otimes \mathscr{B}\left(C^{n}\right)$ to $N \otimes$ $\mathscr{B}\left(C^{n}\right)$ is positive where $t$ is any transposition of matrices relative to any fixed orthonormal basis. The positivity of $F \otimes t$ does not depend on $t$ because two transpositions $t$ and $t^{\prime}$ relative to different orthonormal bases are always related by $t^{\prime}(Q)=u t(Q) u^{*}$ for some unitary $u \in \mathscr{B}\left(C^{n}\right)$.

If $F$ is $n$-positive or transposed- $n$-positive, then $Q \geqq 0$ implies $Q \otimes 1$ $\geqq 0$ and hence $F(Q) \otimes 1 \geqq 0$ and hence $F(Q) \geqq 0$. (More generally it is $n^{\prime}$-positive or transposed- $n^{\prime}$-positive for $n^{\prime} \leqq n$.) Considering $F\left((z+Q)^{*}\right.$ $(z+Q)) \geqq 0$ for $z=1$ and $i$, we then have the selfadjointness $F(Q)^{*}=$ $F\left(Q^{*}\right)$.

Lemma 4. If a linear map $F$ from $M$ into $N$ is 2-positive and satisfies $F(1) F(Q)=F(Q), Q \in M$, then

$$
F\left(Q^{*} Q\right) \geqq F(Q)^{*} F(Q), \quad Q \in M
$$


If a linear map $F$ from $M$ into $N$ is transposed-2-positive and satisfies $F(1) F(Q)=F(Q), Q \in M$, then

$$
F\left(Q^{*} Q\right) \geqq F(Q) F(Q)^{*}, \quad Q \in M .
$$

Proof. Consider

$$
\hat{Q}=\left(\begin{array}{ll}
1 & Q \\
Q^{*} & Q^{*} Q
\end{array}\right) \in M \otimes \mathscr{B}\left(C^{2}\right)
$$

for $Q \in M$ relative to a fixed orthonormal basis $e_{1}$ and $e_{2}$ in $C^{2}$. Let $x_{1}$ and $x_{2}$ be vectors in defining Hilbert space of $M$ and $N$ and $x=x_{1} \otimes e_{1}$ $+x_{2} \otimes e_{2}$. Then

$$
(x, \hat{Q} x)=\left\|x_{1}+Q x_{2}\right\|^{2} \geqq 0
$$

and hence $\hat{Q} \geqq 0$.

If $F$ is 2 -positive then

$$
0 \leqq(x,(F \otimes 1)(\hat{Q}) x)=\left(x_{1}, F(1) x_{1}\right)+2 \operatorname{Re}\left(x_{1}, F(Q) x_{2}\right)+\left(x_{2}, F\left(Q^{*} Q\right) x_{2}\right)
$$

where we have used $F(Q)^{*}=F\left(Q^{*}\right)$. Setting $x_{1}=-F(Q) x_{2}$, we have

$$
0 \leqq\left(x_{2}, F\left(Q^{*} Q\right) x_{2}\right)-\left(x_{2}, F(Q) * F(Q) x_{2}\right)
$$

for any $x_{2}$ where we have used $F(1) F(Q)=F(Q)$. Hence we have (1.1).

If $F$ is transposed-2-positive, we have

$$
0 \leqq(x,(F \otimes t)(\hat{Q}) x)=\left(x_{1}, F(1) x_{1}\right)+2 \operatorname{Re}\left(x_{1}, F(Q) * x_{2}\right)+\left(x_{2}, F\left(Q^{*} Q\right) x_{2}\right)
$$

Hence, by setting $x_{1}=-F(Q) * x_{2}$, we obtain (1.2). $\quad$ Q.E.D.

For a cyclic and separating vector $\Omega$ for $M$, the polar decomposition

$$
\bar{S}=J_{\Omega} \Delta_{\Omega}^{1 / 2}
$$

of the closure $\bar{S}$ of the operator $S$ defined on $M \Omega$ by

$$
S Q \Omega=Q^{*} \Omega, \quad Q \in M
$$

defines the modular operator $\Delta_{\Omega}$, which is a strictly positive selfadjoint 
operator satisfying $\Delta_{\Omega} \Omega=\Omega$ and $J_{\Omega} \Delta_{\Omega}=\Delta_{\Omega}^{-1} J_{\Omega}$, and the modular conjugation $J_{\Omega}$ which is an antiunitary involution satisfying $J_{\Omega} \Omega=\Omega$.

If $\Omega$ is not a cyclic and separating vector, we consider the restrictions of $M$ and $M^{\prime}$ to $s^{M}(\Omega) s^{M^{\prime}}(\Omega) H$, and define $J_{\Omega}$ and $\Delta_{\Omega}$ on $s^{M}(\Omega) s^{M^{\prime}}$ $(\Omega) H$ as above and 0 on $\left(1-s^{M}(\Omega) s^{M^{\prime}}(\Omega)\right) H$. The mapping

$$
\tau_{\Omega}(t) Q \equiv \Delta_{\Omega}^{i t} Q \Delta_{\Omega}^{-i t} s^{M}(\Omega) s^{M^{\prime}}(\Omega)
$$

maps $M$ onto $s^{M^{\prime}}(\Omega) s^{M}(\Omega) M s^{M}(\Omega)$ and $M^{\prime}$ onto $s^{M}(\Omega) s^{M^{\prime}}(\Omega) M^{\prime} s^{M^{\prime}}(\Omega)$. It is an automorphism of $s^{M^{\prime}}(\Omega) s^{M}(\Omega) M s^{M}(\Omega)$ and $s^{M}(\Omega) s^{M^{\prime}}(\Omega) M^{\prime} s^{M^{\prime}}(\Omega)$.

We denote

$$
j_{\Omega}(Q)=J_{\Omega} Q J_{\Omega}
$$

It brings $M$ onto $s^{M^{\prime}}(\Omega) M^{\prime} s^{M^{\prime}}(\Omega) s^{M}(\Omega)$ and $M^{\prime}$ onto $s^{M}(\Omega) M s^{M}(\Omega) s^{M^{\prime}}(\Omega)$.

For a normal positive linear functional $\rho$ on $M$, we denote $J_{\Omega}, \Delta_{\Omega}$, $\tau_{\Omega}(t), j_{\Omega}$ for $\pi_{\rho}(M)$ and $\Omega=\Omega_{\rho}$ by $J_{\rho}, \Delta_{\rho}, \tau_{\rho}(t)$ and $j_{\rho}$. We sometimes denote the expectation functional of $B\left(H_{\rho}\right)$ by the vector $\Omega_{\rho}$ again by $\rho$.

We need the following.

Lemma 5. Let $\rho$ be a normal positive linear functional of $M$ and $Z_{\rho}$ be the set of $x \in M$ such that $\rho(x Q)=\rho(Q x)$ for all $Q \in M$. Then for every $z \in Z_{\rho},\left[s^{M}(\rho), z\right]=0,\left[\Delta_{\rho}, \pi_{\rho}(z)\right]=0$ and

$$
\tau_{\rho}(t) \pi_{\rho}(z)=\pi_{\rho}\left(z s^{M}(\rho)\right)
$$

If $z \in M \cap M^{\prime}$, then

$$
j_{\rho}\left(\pi_{\rho}(z)\right)=\pi_{\rho}\left(z^{*} s^{M}(\rho)\right)
$$

Proof. Substituting $Q s^{M}(\rho)^{\perp}$ into $Q$ of $\rho(x Q)=\rho(Q x)$, we obtain $\rho\left(Q s^{M}(\rho)^{\perp} x\right)=0 \quad$ where $s^{M}(\rho)^{\perp}=1-s^{M}(\rho)$. Hence $\pi_{\rho}\left(s^{M}(\rho)^{\perp} x\right) \Omega_{\rho}=0$. Multiplying $\pi_{\rho}(M)^{\prime}$, we obtain $0=\pi_{\rho}\left(s^{M}(\rho)^{\perp} x\right) s^{\pi_{\rho}(M)}\left(\Omega_{\rho}\right)=\pi_{\rho}\left(s^{M}(\rho)^{\perp} x s^{M}\right.$ ( $\rho)$ ). Substituting $s^{M}(\rho)^{\perp} Q$ into $Q$ of $\rho(x Q)=\rho(Q x)$, we also obtain $\pi_{\rho}\left(s^{M}(\rho) x s^{M}(\rho)^{\perp}\right)=\pi_{\rho}\left(s^{M}(\rho)^{\perp} x^{*} s^{M}(\rho)\right)^{*}=0$. Hence $\pi_{\rho}\left(\left[x, s^{M}(\rho)\right]\right)=0$. Hence $s_{c}(\rho)\left[x, s^{M}(\rho)\right]=0$ where $s_{c}(\rho)$ is the central support of $\rho$. Since $\left[1-s_{c}(\rho)\right] s^{M}(\rho)=0$, we have $\left[x, s^{M}(\rho)\right]=0$. 
Since $\Omega_{\rho}$ is cyclic for $R \equiv \pi_{\rho}(M), s^{R^{\prime}}\left(\Omega_{\rho}\right)=1$. Since $\tau_{\rho}(t) \pi_{\rho}(z)=$ $\tau_{\rho}(t) \pi_{\rho}\left(z s^{M}(\rho)\right)$ and $j_{\rho}\left(\pi_{\rho}(z)\right)=j_{\rho}\left(\pi_{\rho}(z) s^{M}(\rho)\right)$ by definitions of $\tau_{\rho}$ and $j_{\rho}$, it is enough to prove

$$
\begin{aligned}
& \tau_{\rho}(t) \pi_{\rho}(z)=\pi_{\rho}(z) \\
& j_{\rho}\left(\pi_{\rho}(z)\right)=\pi_{\rho}(z)^{*}
\end{aligned}
$$

for $z \in Z_{\rho} s^{M}(\rho)$ on $\pi_{\rho}\left(s^{M}(\rho)\right) H_{\rho} \equiv H_{\rho}^{\prime}$. Since $\Omega_{\rho}$ is cyclic and separating for $R_{\rho} \equiv \pi_{\rho}\left(s^{M}(\rho) M s^{M}(\rho)\right)$ on $H_{\rho}^{\prime}$, the first equation is known. [8] It implies $\left[\Delta_{\rho}, \pi_{\rho}(z)\right]=0$. From $j_{\rho}(\bar{z}) \Omega_{\rho}=\Delta_{\rho}^{1 / 2} \bar{z}^{*} \Omega_{\rho}=\bar{z}^{*} \Omega_{\rho}$ we have $j_{\rho}(\bar{z})=\bar{z}^{*}$ for $\bar{z}=\pi_{\rho}(z), z \in M \cap M^{\prime} s^{M}(\rho)$.

Q.E.D.

\section{§ 2. Mapping $F_{\rho}^{N M}$ from a von Neumann Algebra $M$ into $M^{\prime}$}

Theorem 1. Let $M$ and $N$ be von Neumann algebras such that $N C$ $M^{\prime}$. Let $\rho$ be a normal positive linear functional of $(M \cup N)^{\prime \prime}$. There exists a unique mapping $F_{\rho}^{N M}$ from $M$ into $N$ satisfying

$$
\rho\left(Q Q^{\prime}\right)=\rho\left(F_{\rho}^{N M}(Q) Q^{\prime}+Q^{\prime} F_{\rho}^{N M}(Q)\right) / 2
$$

for all $Q \in M, Q^{\prime} \in N$, and

$$
s\left(F_{\rho}^{N M}(Q)\right) \leqq s^{N}(\rho)
$$

It has the following properties:

(1) $F_{\rho}^{N M}$ is $(M \cap N)$-linear. $F_{\rho}^{N M}(Q)^{*}=F_{\rho}^{N M}\left(Q^{*}\right)$.

(2) $\quad F_{\rho}^{N M}(1)=s^{N}(\rho)$.

(3) $F_{\rho}^{N M}$ is transposed-n-positive for all positive integers $n$. (In particular, $F_{\rho}^{N M}$ is positive and $\left.F_{\rho}^{N M}(Q)^{*}=F_{\rho}^{N M}\left(Q^{*}\right)_{\text {。 }}\right)$

(4) $\left\|F_{\rho}^{N M}\right\|=1$ for $\rho \neq 0$.

(5) $F_{\rho}^{N M}$ is $\sigma$-weakly continuous (i.e. normal). It is continuous on the unit ball relative to the strong topology on $M$ and * strong topology on $N$. 
(6) For any automorphism $\tau$ of $(M \cup N)^{\prime \prime}$ satisfying $\tau(M)=M$ and $\tau(N)=N$,

$$
F_{\rho}^{N M}(\tau Q)=\tau F_{\rho * \tau}^{N M}(Q)
$$

where $\tau^{*} \rho$ is defined by $\left(\tau^{*} \rho\right)(Q)=\rho(\tau Q)$. In particular, if $u \in M$ is unitary,

$$
F_{\rho}^{N M}\left(u Q u^{*}\right)=F_{u^{*} \rho u}^{N M}(Q)
$$

and if $v \in N$ is unitary

$$
v F_{\rho}^{N M}(Q) v^{*}=F_{v \rho v^{*}}^{N M}(Q)
$$

where $\left(t_{1} \rho t_{2}\right)(Q)=\rho\left(t_{2} Q t_{1}\right)$.

(7) For any $A \in M \cap N, A \geqq 0$,

$$
F_{A \rho}^{N M}(Q)=F_{\rho}^{N M}(Q) s(A) .
$$

(8) If $\lim _{n}\left\|\rho_{n}-\rho\right\|=0$ and $\lim _{n} s^{N}\left(\rho_{n}\right)=s^{N}(\rho)$, then

$$
\lim _{n} F_{\rho_{n}}^{N M}(Q)=F_{\rho}^{N M}(Q), \lim _{n} F_{\rho_{n}}^{N M}(Q)^{*}=F_{\rho}^{N M}(Q)^{*}
$$

uniformly for a bounded set of $Q$. (If $s^{N}\left(\rho_{n}\right) \leqq s^{N}(\rho)$, then $\lim _{n}\left\|\rho_{n}-\rho\right\|$ $=0$ implies $\lim s^{N}\left(\rho_{n}\right)=s^{N}(\rho)$.)

Proof. Let $Q \in M$ and $Q^{\prime} \in N$. Consider

$$
f_{Q}\left(Q^{\prime}\right)=\rho\left(Q Q^{\prime}\right)
$$

If $Q \geqq 0$, then

$$
f_{Q}\left(Q^{\prime}\right)=\rho\left(Q^{1 / 2} Q^{\prime} Q^{1 / 2}\right)
$$

is normal positive linear functional on $N$. If $Q^{\prime} \geqq 0$ in addition,

$$
f_{Q}\left(Q^{\prime}\right)=\rho\left(Q^{\prime 1 / 2} Q Q^{\prime 1 / 2}\right) \leqq\|Q\| \rho\left(Q^{\prime}\right)
$$

Hence $f_{Q} \leqq\|Q\| \rho$. 
For general $Q$, we have

$$
Q=Q_{1}-Q_{2}+i\left(Q_{3}-Q_{4}\right)
$$

where $Q_{1}$ and $Q_{2}$ are positive and negative parts of $\left(Q+Q^{*}\right) / 2, Q_{3}$ and $Q_{4}$ are positive and negative parts of $\left(Q-Q^{*}\right) /(2 i)$. Then

$$
f_{Q}=f_{Q_{1}}-f_{Q_{2}}+i\left(f_{Q_{3}}-f_{Q_{4}}\right)
$$

where $f_{Q_{k}} \leqq\left\|Q_{k}\right\| \rho$.

By Lemma 3, there exists a unique $h_{0}=F_{\rho}^{N M}(Q) \in N$ such that

$$
f_{Q}\left(Q^{\prime}\right)=\rho\left(F_{\rho}^{N M}(Q) Q^{\prime}+Q^{\prime} F_{\rho}^{N M}(Q)\right) / 2
$$

for all $Q^{\prime} \in N$ and

$$
s\left(F_{\rho}^{N M}(Q)\right) \leqq s^{N}(\rho)
$$

This shows the existence and uniqueness of $F_{\rho}^{N M}$.

(1) Let $z_{1}, z_{2} \in M \cap N$ and $Q_{1}, Q_{2} \in M$. Note that $M \cap N$ is in the center of $(N \cup M)^{\prime \prime}$ by $N \subset M^{\prime}$. We have, for $Q=z_{1} Q_{1}+z_{2} Q_{2}$,

$$
\begin{aligned}
\rho\left(F_{\rho}^{N M}(Q) Q^{\prime}+Q^{\prime} F_{\rho}^{N M}(Q)\right) / 2=\rho\left(Q Q^{\prime}\right) \\
=\rho\left(Q_{1} z_{1} Q^{\prime}\right)+\rho\left(Q_{2} z_{2} Q^{\prime}\right) \\
=\rho\left(F_{\rho}^{N M}\left(Q_{1}\right) z_{1} Q^{\prime}+z_{1} Q^{\prime} F_{\rho}^{N M}\left(Q_{1}\right)\right) / 2 \\
\quad+\rho\left(F_{\rho}^{N M}\left(Q_{2}\right) z_{2} Q^{\prime}+z_{2} Q^{\prime} F_{\rho}^{N M}\left(Q_{2}\right)\right) / 2 \\
=\rho\left(F^{\prime} Q^{\prime}+Q^{\prime} F^{\prime}\right) / 2
\end{aligned}
$$

where

$$
F^{\prime}=z_{1} F_{\rho}^{N M}\left(Q_{1}\right)+z_{2} F_{\rho}^{N M}\left(Q_{2}\right)
$$

Since $s\left(F_{\rho}^{N M}\left(Q_{k}\right)\right) \leqq s^{N}(\rho), k=1,2$, we also have $s\left(F^{\prime}\right) \leqq s^{N}(\rho)$. By the uniqueness, we have

$$
F^{\prime}=F_{\rho}^{N M}\left(z_{1} Q_{1}+z_{2} Q_{2}\right) .
$$


From $\rho\left(Q^{*} Q^{\prime}\right)=\rho\left(Q\left(Q^{\prime}\right)^{*}\right)^{*}$ and the uniqueness, we obtain $F_{\rho}^{N M}(Q)^{*}=F_{\rho}^{N M}\left(Q^{*}\right)$.

(2) The substitution of $Q=1$ and $F_{\rho}^{N M}(Q)=s^{N}(\rho)$ into (2.1) and (2.2) immediately prove this statement.

(3) If $Q \geqq 0$, then $F_{\rho}^{N M}(Q) \geqq 0$ from Lemma 1 . Hence $F_{\rho}^{N M}$ is positive.

To prove transposed- $n$-positivity for $n>1$, let $e_{1}, \ldots, e_{n}$ be an orthonormal basis of $C^{n}$,

$$
\Omega=n^{-1 / 2} \sum_{k=1}^{n} e_{k} \otimes e_{k} \in C^{n} \otimes C^{n}
$$

$J_{\Omega}$ be the modular conjugation for $\Omega\left(J_{\Omega} \sum c_{i j} e_{i} \otimes e_{j}=\sum \bar{c}_{i j} e_{j} \otimes e_{i}\right)$, and the transposition $t$ be chosen to be

$$
{ }^{t} Q=J_{\Omega} Q^{*} J_{\Omega}
$$

which maps $Q \in \mathscr{B}\left(C^{n}\right) \otimes 1$ onto $1 \otimes \mathscr{B}\left(C^{n}\right)$. Consider (on $H \otimes\left(C^{n} \otimes C^{n}\right)$ )

$$
\begin{aligned}
& \bar{M}=M \otimes\left(\mathscr{B}\left(C^{n}\right) \otimes 1\right), \\
& \bar{N}=N \otimes\left(1 \otimes \mathscr{B}\left(C^{n}\right)\right), \\
& \bar{\rho}=\rho \otimes \omega_{\Omega} .
\end{aligned}
$$

Then $F_{\rho}^{N M} \otimes t$ from $\bar{M}$ to $\bar{N}$ coincides with $F_{\bar{\rho}}^{\bar{N} \bar{M}}$ due to the following computation and hence is positive by our earlier result.

Let $Q_{1} \in M, Q_{1}^{\prime} \in N, Q_{2} \in \mathscr{B}\left(C^{n}\right) \otimes 1, Q_{2}^{\prime} \in 1 \otimes \mathscr{B}\left(C^{n}\right)$. Then

$$
\begin{aligned}
\bar{\rho}\left(\left(Q_{1} \otimes Q_{2}\right)\left(Q_{1}^{\prime} \otimes Q_{2}^{\prime}\right)\right)=\rho\left(Q_{1} Q_{1}^{\prime}\right)\left(\Omega, Q_{2} Q_{2}^{\prime} \Omega\right) \\
=\rho\left(F_{\rho}^{N M}\left(Q_{1}\right) Q_{1}^{\prime}\right)\left(Q_{2}^{*} \Omega, Q_{2}^{\prime} \Omega\right) / 2 \\
\quad+\rho\left(Q_{1}^{\prime} F_{\rho}^{N M}\left(Q_{1}\right)\right)\left(\Omega, Q_{2}^{\prime} Q_{2} \Omega\right) / 2 \\
=\rho\left(F_{\rho}^{N M}\left(Q_{1}\right) Q_{1}^{\prime}\right)\left(j_{\Omega}\left(Q_{2}\right) \Omega, Q_{2}^{\prime} \Omega\right) / 2 \\
\quad+\rho\left(Q_{1}^{\prime} F_{\rho}^{N M}\left(Q_{1}\right)\right)\left(\Omega, Q_{2}^{\prime} j_{\Omega}\left(Q_{2}^{*}\right) \Omega\right) / 2
\end{aligned}
$$

where we have used the fact that the modular operator for a faithful 
trace vector $\Omega$ is 1 and hence $j_{\Omega}(Q) \Omega=J_{\Omega} Q \Omega=\Delta_{\Omega}^{1 / 2} Q^{*} \Omega=Q^{*} \Omega$. Substituting the definition of ${ }^{t} Q$, we have

$$
\begin{aligned}
\bar{\rho}\left(\left(Q_{1} \otimes Q_{2}\right) Q^{\prime}\right)= & \bar{\rho}\left(\left\{F_{\rho}^{N M}\left(Q_{1}\right) \otimes^{t} Q_{2}\right\} Q^{\prime}\right) / 2 \\
& +\bar{\rho}\left(Q^{\prime}\left\{F_{\rho}^{N M}\left(Q_{1}\right) \otimes^{t} Q_{2}\right\}\right) / 2
\end{aligned}
$$

for $Q^{\prime}=Q_{1}^{\prime} \otimes Q_{2}^{\prime}$. Since such $Q^{\prime}$ linearly span $N \otimes\left(1 \otimes \mathscr{B}\left(C^{n}\right)\right)$, the same equation holds for all $Q^{\prime}$ in $\bar{N}$. Since $s^{\bar{N}}(\bar{\rho})=s^{N}(\rho) \otimes 1$ because $\Omega$ is cyclic for $1 \otimes \mathscr{B}\left(C^{n}\right)$, we have $s\left(F_{\rho}^{N M}\left(Q_{1}\right) \otimes^{t} Q_{2}\right) \leqq s\left(F_{\rho}^{N M}\left(Q_{1}\right)\right) \otimes 1 \leqq s^{\bar{N}}(\bar{\rho})$. Hence

$$
F_{\bar{\rho}}^{\bar{N}} \bar{M}\left(Q_{1} \otimes Q_{2}\right)=\left(F_{\rho}^{N M} \otimes t\right)\left(Q_{1} \otimes Q_{2}\right)
$$

(4) From Lemma 1 (3) and (2.7), we have

$$
\left\|F_{\rho}^{N M}(Q)\right\| \leqq\|Q\|
$$

for $Q \geqq 0$. Due to Lemma 4 , we have

$$
\begin{aligned}
\left\|F_{\rho}^{N M}(Q)\right\|^{2} & =\left\|F_{\rho}^{N M}(Q) F_{\rho}^{N M}(Q)^{*}\right\| \\
& \leqq\left\|F_{\rho}^{N M}\left(Q^{*} Q\right)\right\| \leqq\left\|Q^{*} Q\right\|=\|Q\|^{2}
\end{aligned}
$$

for arbitrary $Q$. From (2), we obtain $\left\|F_{\rho}^{N M}\right\|=1$ if $\rho \neq 0$.

(5) Assume that a net $Q_{\alpha} \in M$ has a weak limit $Q$ and $\left\|Q_{\alpha}\right\| \leqq 1$. Then

$$
\lim _{\alpha} \rho\left(F_{\rho}^{N M}\left(Q_{\alpha}\right) Q^{\prime}+Q^{\prime} F_{\rho}^{N M}\left(Q_{\alpha}\right)\right)=\rho\left(F_{\rho}^{N M}(Q) Q^{\prime}+Q^{\prime} F_{\rho}^{N M}(Q)\right)
$$

Since $\left\|F_{\rho}^{N M}\left(Q_{\alpha}\right)\right\| \leqq\left\|Q_{\alpha}\right\| \leqq 1$, the set of accumulation points

$$
\bigcap_{\beta}\left(\bigcup_{\alpha>\beta} F_{\rho}^{N M}\left(Q_{\alpha}\right)\right)^{-(\text {weak })}
$$

is non-empty due to the weak compactness. Let $\bar{Q}$ be in this set. Then from (2.13), we have

$$
\rho\left(F_{\rho}^{N M}(Q) Q^{\prime}+Q^{\prime} F_{\rho}^{N M}(Q)\right)=\rho\left(\bar{Q} Q^{\prime}+Q^{\prime} \bar{Q}\right) .
$$

From the uniqueness in Lemma 2, we have 


$$
\bar{Q}=F_{\rho}^{N M}(Q)
$$

and hence the set (2.14) consists of a single point $F_{\rho}^{N M}(Q)$. Thus

$$
\mathrm{w}-\lim _{\alpha} F_{\rho}^{N M}\left(Q_{\alpha}\right)=F_{\rho}^{N M}\left(\mathrm{w}-\lim _{\alpha} Q_{\alpha}\right) .
$$

The weak continuity on bounded sets implies the normality and the $\sigma$-weak continuity for a positive linear mapping.

Next, we assume that a net $Q_{\alpha} \in M$ has a strong limit $Q$ and $\left\|Q_{\alpha}\right\|$ $\leqq 1$. Then $\left\|F_{\rho}^{N M}\left(Q_{\alpha}-Q\right)\right\| \leqq\left\|Q_{\alpha}-Q\right\| \leqq 2$. Hence

$$
\lim _{\alpha} \rho\left(\left\{F_{\rho}^{N M}\left(Q_{\alpha}-Q\right)\right\}^{*}\left(Q_{\alpha}-Q\right)\right)=0
$$

By using (2.1) with $Q=\left(Q_{\alpha}-Q\right)^{*}, Q^{\prime}=F_{\rho}^{N M}\left(Q_{\alpha}-Q\right)$, we have

$$
\begin{aligned}
0 \leqq & \left(\left\{F_{\rho}^{N M}\left(Q_{\alpha}-Q\right)\right\}^{*} F_{\rho}^{N M}\left(Q_{\alpha}-Q\right)\right)+\rho\left(F_{\rho}^{N M}\left(Q_{\alpha}-Q\right)\left\{F_{\rho}^{N M}\left(Q_{\alpha}-Q\right)\right\}^{*}\right) \\
& =2 \rho\left(\left\{F_{\rho}^{N M}\left(Q_{\alpha}-Q\right)\right\}^{*}\left(Q_{\alpha}-Q\right)\right) \rightarrow 0
\end{aligned}
$$

and hence

$$
\begin{aligned}
& \lim \pi_{\rho}\left\{F_{\rho}^{N M}\left(Q_{\alpha}-Q\right)\right\} \Omega_{\rho}=0 \\
& \lim \pi_{\rho}\left\{F_{\rho}^{N M}\left(Q_{\alpha}-Q\right)\right\}^{*} \Omega_{\rho}=0
\end{aligned}
$$

Multiplying $\hat{Q} \in \pi_{\rho}(N)^{\prime}$, we have

$$
\begin{aligned}
& \lim \pi_{\rho}\left\{F_{\rho}^{N M}\left(Q_{\alpha}-Q\right)\right\} \Psi=0, \\
& \lim \pi_{\rho}\left\{F_{\rho}^{N M}\left(Q_{\alpha}-Q\right)^{*}\right\} \Psi=0 .
\end{aligned}
$$

for $\Psi=\hat{Q} \Omega_{\rho}$. Since $\left\|F_{\rho}^{N M}\left(Q_{\alpha}-Q\right)\right\| \leqq 2$, the same hold on the closure of $\pi_{\rho}(N)^{\prime} \Omega_{\rho}$, which is $\pi_{\rho}\left(s^{N}(\rho)\right) H_{\rho}$. Hence

$\lim \pi_{\rho}\left\{F_{\rho}^{N M}\left(Q_{\alpha}-Q\right) s^{N}(\rho)\right\}=0, \quad \lim \pi_{\rho}\left\{F_{\rho}^{N M}\left(Q_{\alpha}-Q\right) * s^{N}(\rho)\right\}=0$.

Since $\pi_{\rho}$ is faithful at least on $s^{N}(\rho) N s^{N}(\rho), \pi_{\rho}^{-1}$ is continuous on $s^{N}(\rho) N s^{N}(\rho)$ and

$$
0=\lim _{\alpha} s^{N}(\rho) F_{\rho}^{N M}\left(Q_{\alpha}-Q\right) s^{N}(\rho)=\lim _{\alpha}\left\{F_{\rho}^{N M}\left(Q_{\alpha}\right)-F_{\rho}^{N M}(Q)\right\},
$$




$$
0=\lim _{\alpha} s^{N}(\rho) F_{\rho}^{N M}\left(Q_{\alpha}-Q\right)^{*} s^{N}(\rho)=\lim _{\alpha}\left\{F_{\rho}^{N M}\left(Q_{\alpha}\right)^{*}-F_{\rho}^{N M}(Q)^{*}\right\}
$$

due to (2.2) and (1).

(6) For $Q \in M$ and $Q^{\prime} \in N$, we have

$$
\begin{aligned}
\rho\left(\tau(Q) Q^{\prime}\right) & =\rho\left(\tau\left\{Q \tau^{-1} Q^{\prime}\right\}\right) \\
& =\tau^{*} \rho\left(Q \tau^{-1} Q^{\prime}\right) \\
& =\tau^{*} \rho\left(F_{\tau^{*} \rho}^{N M}(Q) \tau^{-1} Q^{\prime}\right) / 2+\tau^{*} \rho\left(\left\{\tau^{-1} Q^{\prime}\right\} F_{\tau^{*} \rho}^{N M}(Q)\right) / 2 \\
& =\rho\left(\left\{\tau F_{\tau^{*} \rho}^{N M}(Q)\right\} Q^{\prime}\right) / 2+\rho\left(Q^{\prime} \tau F_{\tau^{*} \rho}^{N M}(Q)\right) / 2 .
\end{aligned}
$$

We also have

$$
\begin{aligned}
s\left(\tau F_{\tau * \rho}^{N M}(Q)\right) & =\tau\left\{s\left(F_{\tau * \rho}^{N M}(Q)\right)\right\} \\
& \leqq \tau\left\{s^{N}\left(\tau^{*} \rho\right)\right\}=s^{N}(\rho) .
\end{aligned}
$$

Hence (2.3) holds by the uniqueness.

(2.4) and (2.5) are special cases of (2.3) where $\tau(A)=u A u^{*}$ and $\tau(A)=v A v^{*}$ for $A \in(N \cup M)^{\prime \prime}$.

(7) Since $N \subset M^{\prime}, M \cap N$ is in the center of $(N \cup M)^{\prime \prime}$. We have

$$
\begin{aligned}
(A \rho)\left(Q Q^{\prime}\right) & =\rho\left(Q Q^{\prime} A\right) \\
& =\rho\left(F_{\rho}^{N M}(Q) Q^{\prime} A\right) / 2+\rho\left(Q^{\prime} A F_{\rho}^{N M}(Q)\right) / 2 \\
& =A \rho\left(s(A) F_{\rho}^{N M}(Q) Q^{\prime}+Q^{\prime} s(A) F_{\rho}^{N M}(Q)\right) / 2 .
\end{aligned}
$$

We also have

$$
s\left\{s(A) F_{\rho}^{N M}(s)\right\}=s(A) s\left(F_{\rho}^{N M}(s)\right) \leqq s(A) s^{N}(\rho)=s^{N}(A \rho)
$$

Hence, by uniqueness, we have

$$
F_{A \rho}^{N M}(Q)=F_{\rho}^{N M}(Q) s(A)
$$

(8) We have for $\delta_{n} \equiv F_{\rho}^{N M}(Q)-F_{\rho_{n}}^{N M}(Q)$ the following estimate $\left|\rho\left(\delta_{n} Q^{\prime}+Q^{\prime} \delta_{n}\right)\right| \leqq 2\left|\rho\left(Q Q^{\prime}\right)-\rho_{n}\left(Q Q^{\prime}\right)\right|$ 


$$
\begin{aligned}
& +\left|\rho_{n}\left(F_{\rho_{n}}^{N M}(Q) Q^{\prime}+Q^{\prime} F_{\rho_{n}}^{N M}(Q)\right)-\rho\left(F_{\rho_{n}}^{N M}(Q) Q^{\prime}+Q^{\prime} F_{\rho_{n}}^{N M}(Q)\right)\right| \\
\leqq & 4\|Q\|\left\|Q^{\prime} \mid\right\|\left\|\rho-\rho_{n}\right\| .
\end{aligned}
$$

Setting $Q^{\prime}=\delta_{n}^{*}$ and using $\left\|\delta_{n}\right\| \leqq 2\|Q\|$, we have

$$
\begin{aligned}
& 0 \leqq \rho\left(\delta_{n}^{*} \delta_{n}\right) \leqq \rho\left(\delta_{n} \delta_{n}^{*}+\delta_{n}^{*} \delta_{n}\right) \leqq 8\|Q\|^{2}\left\|\rho-\rho_{n}\right\| \\
& 0 \leqq \rho\left(\delta_{n} \delta_{n}^{*}\right) \leqq 8\|Q\|^{2}\left\|\rho-\rho_{n}\right\| .
\end{aligned}
$$

Hence we have

$$
\lim _{n \rightarrow \infty} \pi_{\rho}\left(\delta_{n}\right) \Psi=0, \quad \lim _{n \rightarrow \infty} \pi_{\rho}\left(\delta_{n}^{*}\right) \Psi=0
$$

for $\Psi=\Omega_{\rho}$ and hence for $\Psi=Q^{\prime} \Omega_{\rho}, Q^{\prime} \in \pi_{p}(N)^{\prime}$. Since $\left\|\pi_{\rho}\left(\delta_{n}\right)\right\| \leqq 2\|Q\|$ is uniformly bounded, the same holds for $\Psi \in s^{N}\left(\Omega_{\rho}\right) H_{\rho}$ and hence

$$
\lim _{n \rightarrow \infty} \pi_{\rho}\left(\delta_{n} s^{N}(\rho)\right)=0, \quad \lim _{n \rightarrow \infty} \pi_{\rho}\left(\delta_{n}^{*} s^{N}(\rho)\right)=0
$$

uniformly for a bounded set of $Q$. Since $\pi_{\rho}^{-1}$ is continuous on $N s_{c}^{N}(\rho)$, where $s_{c}^{N}(\rho)$ is the central support of $s^{N}(\rho)$, we have

$$
\begin{aligned}
& \lim _{n \rightarrow \infty}\left\{F_{\rho}^{N M}(Q)-F_{\rho_{n}}^{N M}(Q) s^{N}(\rho)\right\}=0, \\
& \lim _{n \rightarrow \infty}\left\{F_{\rho}^{N M}(Q)^{*}-F_{\rho_{n}}^{N M}(Q)^{*} s^{N}(\rho)\right\}=0 .
\end{aligned}
$$

If $\lim s^{N}\left(\rho_{n}\right)=s^{N}(\rho)$, then as $\left\|F_{\rho_{n}}^{N M}(Q)\right\| \leqq\|Q\|$ we have

$$
\begin{aligned}
\lim _{n \rightarrow \infty} & \left\{F_{\rho_{n}}^{N M}(Q) s^{N}(\rho)-F_{\rho_{n}}^{N M}(Q)\right\} \\
= & \lim _{n \rightarrow \infty} F_{\rho_{n}}^{N M}(Q)\left(s^{N}(\rho)-s^{N}\left(\rho_{n}\right)\right)=0
\end{aligned}
$$

and we obtain

$$
\lim F_{\rho_{n}}^{N M}(Q)=\lim F_{\rho}^{N M}(Q)
$$

uniformly for a bounded set of $Q$. Similar equation for adjoint also holds. If $s^{N}\left(\rho_{n}\right) \leqq s^{N}(\rho)$, then

$$
\left|\rho\left(1-s^{N}\left(\rho_{n}\right)\right)\right|=\left|\rho\left(1-s^{N}\left(\rho_{n}\right)\right)-\rho_{n}\left(1-s^{N}\left(\rho_{n}\right)\right)\right| \leqq\left\|\rho-\rho_{n}\right\|
$$


and hence

$$
\lim \pi_{\rho}\left(1-s^{N}\left(\rho_{n}\right)\right) \Omega_{\rho}=0 .
$$

As before, we have

$$
\lim \left(s^{N}(\rho)-s^{N}\left(\rho_{n}\right)\right)=0 .
$$

The proof of (3) implies the following corollaries.

Corollary 1. If $M^{\prime}=N, \rho=\omega_{\Omega}$ and $\Omega$ is a faithful trace vector for $M$ as well as for $N$, then

$$
F_{\rho}^{N M}(Q)=J_{\Omega} Q^{*} J_{\Omega}
$$

Corollary 2. Let $M=M_{1} \otimes M_{2}, N=N_{1} \otimes N_{2}, \rho=\rho_{1} \otimes \rho_{2}$. If $\rho_{1}$ is a trace on $N_{1}$ or if $\rho_{2}$ is a trace on $N_{2}$, then

$$
F^{N M}\left(Q_{1} \otimes Q_{2}\right)=F_{\rho_{1}}^{N_{1}}\left(Q_{1}\right) \otimes F_{\rho_{2}}^{N_{2}}\left(Q_{2}\right)
$$

for all $Q_{1} \in M_{1}, Q_{2} \in M_{2}$. (In particular, if either $N_{1}$ or $N_{2}$ is abelian then this holds for any normal states $\rho_{1}$ and $\rho_{2}$.)

Remark 1. $F_{\rho_{M}}^{N M}(Q)=F_{\rho}^{N N^{\prime}}(Q)$ for $Q \in M$, where $\rho_{M}$ is the restriction of $\rho$ (which is a functional on $\left.\left(N \cup N^{\prime}\right)^{\prime \prime}\right)$ to $(M \cup N)^{\prime \prime}$. In this sense, the case $M=N^{\prime}$ is most canonical and we shall study it from different viewpoint in the next section.

Remark 2. In order to define $F_{\rho}^{N M}(Q), \rho$ need not be normal on the whole $(M \cup N)^{\prime \prime}$, but it is sufficient that $\rho$ is normal on $N$. The uniqueness and existence together with properties (1), (2), (3), (4), (6), (7) and (8) hold for such non-normal $\rho$. Note that $f_{Q}$ defined by $(2.6)$ is normal due to (2.7) if $\rho$ is normal on $N$.

Remark 3. Theorem 1 holds also for the case where $N$ is a weakly closed * subalgebra of $M^{\prime}$ even if the unit in $N$ is not the identity operator in $M^{\prime}$. 


\section{§3. Mapping $G_{\rho}^{M}$ from a von Neumann Algebra $M$ into Itself}

Theorem 2. Let $\rho$ be a normal positive linear functional of $M$. There exists a unique mapping $G_{\rho}^{M}$ from $M$ into $s^{M}(\rho) M s^{M}(\rho)$ satisfying

$$
\left(\Omega_{\rho}, \pi_{\rho}(Q) \Delta_{\rho}^{1 / 2} \pi_{\rho}\left(Q^{\prime}\right) \Omega_{\rho}\right)=\rho\left(G_{\rho}^{M}(Q) Q^{\prime}+Q^{\prime} G_{\rho}^{M}(Q)\right) / 2
$$

for all $Q, Q^{\prime} \in M$.

It has the following properties:

(1) $G_{\rho}^{M}$ is $Z_{\rho}$-linear, where $Z_{\rho}$ is the set of $x \in M$ such that $\rho(x Q)=$ $\rho(Q x)$ for all $Q \in M$, and $M$ is considered as two-sided $Z_{\rho}$ module. In particular, $G_{\rho}^{M}$ is $Z$-linear for the center $Z=M \cap M^{\prime}$.

(2) $G_{\rho}^{M}(1)=s^{M}(\rho)$.

(3) $G_{\rho}^{M}$ is completely positive. (In particular, it is positive and $G^{M}(Q)^{*}=G^{M}\left(Q^{*}\right)$

(4) $\left\|G_{\rho}^{M}\right\|=1$ for $\rho \neq 0$.

(5) $G_{\rho}^{M}$ is $\sigma$-weakly continuous (i.e. normal). It is continuous on the unit ball relative to the strong topology for $Q$ and * strong topology for $G_{\rho}^{M}(Q)$.

(6) If $\tau$ is an automorphism of $M$ and $\tau^{*} \rho=\rho$, then

$$
G_{\rho}^{M}(\tau Q)=\tau G_{\rho}^{M}(Q) .
$$

(7) If $z \in Z, z \geqq 0$, then

$$
G_{z \rho}^{M}(Q)=G^{M}(Q) s^{M}(z) .
$$

(8) The kernel of $G_{\rho}^{M}$ is

$$
s^{M}(\rho) M\left(1-s^{M}(\rho)\right)+\left(1-s^{M}(\rho)\right) M
$$

which implies

$$
G_{\rho}^{M}(Q)=G_{\rho}^{M}\left(s^{M}(\rho) Q s^{M}(\rho)\right) .
$$


The image of $G_{\rho}^{M}$ is strongly dense in $s^{M}(\rho) M s^{M}(\rho)$.

Proof. Let $R=\pi_{\rho}(M)$ and $Q, Q^{\prime} \in R$. From the formula $j_{\Omega}(Q) \Omega=$ $\Delta_{\Omega}^{1 / 2} Q^{*} \Omega$ and (2.1), we have

$$
\begin{gathered}
\left(\Omega_{\rho}, Q \Delta_{\rho}^{1 / 2} Q^{\prime} \Omega_{\rho}\right)=\left(\Omega_{\rho}, Q j_{\rho}\left\{Q^{*}\right\} \Omega_{\rho}\right) \\
=\left(\Omega_{\rho}, F_{\rho}^{R^{\prime} R}(Q) j_{\rho}\left(Q^{*}\right) \Omega_{\rho}\right) / 2 \\
+\left(j_{\rho}\left(Q^{\prime}\right) \Omega_{\rho}, F_{\rho}^{R^{\prime} R}(Q) \Omega_{\rho}\right) / 2
\end{gathered}
$$

where $\rho$ is also used for $\rho(Q)=\left(\Omega_{\rho}, Q \Omega_{\rho}\right), Q \in\left(R \cup R^{\prime}\right)^{\prime \prime}$, in writing $F_{\rho}^{R^{\prime} R}$. Since $\left(J_{\rho} x, y\right)=\left(J_{\rho}^{2} J_{\rho} x, y\right)=\left(J_{\rho} x, J_{\rho}^{2} y\right)=\overline{\left(x, J_{\rho} y\right)}=\left(J_{\rho} y, x\right)$ where $J_{\rho}^{2}=$ $s^{R}\left(\Omega_{\rho}\right)$ is hermitian $\left(s^{R^{\prime}}\left(\Omega_{\rho}\right)=1\right.$ due to the cyclicity of $\left.\Omega_{\rho}\right)$, and since $J_{\rho} \Omega_{\rho}=\Omega_{\rho}$, we have

$$
\begin{aligned}
\left(\Omega_{\rho}, Q \Delta_{\rho}^{1 / 2} Q^{\prime} \Omega_{\rho}\right)= & \left(Q^{*} \Omega_{\rho}, j_{\rho}\left(F_{\rho}^{R^{\prime} R}(Q)^{*}\right) \Omega_{\rho}\right) / 2 \\
& +\left(j_{\rho}\left(F_{\rho}^{R^{\prime} R}(Q)\right) \Omega_{\rho}, Q^{\prime} \Omega_{\rho}\right) / 2 .
\end{aligned}
$$

Since $s^{R}\left(j_{\rho}\left(F_{\rho}^{R^{\prime} R}(Q)\right)\right) \leqq s^{R}\left(\Omega_{\rho}\right)=\pi_{\rho}\left(s^{M}(\rho)\right)$, there exists $G \in s^{M}(\rho)$ $M s^{M}(\rho)$ such that

$$
\pi_{\rho}(G)=j_{\rho}\left(F_{\rho}^{R^{\prime} R}\left(Q^{*}\right)\right)
$$

From (3.2) and (3.3), $G_{\rho}^{M}(Q)=G$ satisfies (3.1) for all $Q^{\prime} \in M$. Hence the existence is proved.

If $G_{\rho}^{M}(Q)=G$ and $G^{\prime}$ both satisfy (3.1), then $G-G^{\prime}$ also satisfies $\rho\left(\left(G-G^{\prime}\right) Q^{\prime}+Q^{\prime}\left(G-G^{\prime}\right)\right)=0$ for all $Q^{\prime} \in M$. In particular, we have $\rho\left(\left(G-G^{\prime}\right)^{*}\left(G-G^{\prime}\right)\right)=0$ for $Q^{\prime}=\left(G-G^{\prime}\right)^{*}$. Since $\rho$ is faithful on $s(\rho) M s(\rho)$, we have $G-G^{\prime}=0$ and hence the uniqueness.

(1) From (3.4) and Theorem 1 (1), $G_{\rho}^{M}$ is linear. If $z \in Z_{\rho}$, then $\bar{z}=\pi_{\rho}(z)$ commutes with $\Delta_{\rho}$ (Lemma 5 ) and we have

$$
\begin{gathered}
\left(\Omega_{\rho}, \bar{Q} \bar{z} \Delta_{\rho}^{1 / 2} \bar{Q}^{\prime} \Omega_{\rho}\right)=\left(\Omega_{\rho}, \bar{Q} \Delta_{\rho}^{1 / 2} \bar{z} \bar{Q}^{\prime} \Omega_{\rho}\right) \\
\quad=\rho\left(G_{\rho}^{M}(Q) z Q^{\prime}+z Q^{\prime} G_{\rho}^{M}(Q)\right) / 2 \\
\quad=\rho\left(G_{\rho}^{M}(Q) z Q^{\prime}+Q^{\prime} G_{\rho}^{M}(Q) z\right) / 2
\end{gathered}
$$


for $\bar{Q}=\pi_{\rho}(Q)$ and $\bar{Q}^{\prime}=\pi_{\rho}\left(Q^{\prime}\right), Q \in M, Q^{\prime} \in M$. Since $z$ commutes with $s(\rho)$ by Lemma $5, s\left(G_{\rho}^{M}(Q) z\right) \leqq s(\rho)$ and hence

$$
G_{\rho}^{M}(Q z)=G_{\rho}^{M}(Q) z .
$$

Since $\left[j_{\rho}(\bar{z}), \Delta_{\rho}\right]=j_{\rho}\left(\left[\bar{z}, \Delta_{\rho}^{-1}\right]\right)=0$, we also have

$$
\begin{aligned}
\left(\Omega_{\rho},\right. & \left.\bar{z} \bar{Q} \Delta_{\rho}^{1 / 2} \bar{Q}^{\prime} \Omega_{\rho}\right)=\left(\bar{z}^{*} \Omega_{\rho}, \bar{Q} \Delta_{\rho}^{1 / 2} \bar{Q}^{\prime} \Omega_{\rho}\right) \\
& =\left(j_{\rho}(\bar{z}) \Omega_{\rho}, \bar{Q} \Delta_{\rho}^{1 / 2} \bar{Q}^{\prime} \Omega_{\rho}\right) \\
& =\left(\Omega_{\rho}, \bar{Q} \Delta_{\rho}^{1 / 2} \bar{Q}^{\prime} j_{\rho}\left(\bar{z}^{*}\right) \Omega_{\rho}\right) \\
& =\left(\Omega_{\rho}, \bar{Q} \Delta_{\rho}^{1 / 2} \bar{Q}^{\prime} \bar{z} \Omega_{\rho}\right) \\
& =\rho\left(G_{\rho}^{M}(Q) Q^{\prime} z+Q^{\prime} z G_{\rho}^{M}(Q)\right) / 2 \\
& =\rho\left(z G_{\rho}^{M}(Q) Q^{\prime}+Q^{\prime} z G_{\rho}^{M}(Q)\right) / 2 .
\end{aligned}
$$

Hence we have

$$
G_{\rho}^{M}(z Q)=z G_{\rho}^{M}(Q)
$$

(2), (4) and (5) follow from the corresponding results in Theorem 1 and (3.4).

(3) Let $Q_{i j} \in R$ such that $\sum_{i, j}\left(x_{i}, Q_{i j} x_{j}\right) \geqq 0$ for any $x_{j} \in H_{\rho}$ where the indices $i, j$ run from 1 to $n$. By Theorem 1 (3),

$$
\sum\left(x_{i}, F_{\rho}^{R^{\prime} R}\left(Q_{j i}\right) x_{j}\right) \geqq 0
$$

for any vectors $x_{j} \in H_{\rho}$. Hence

$$
\begin{aligned}
& \sum\left(x_{i}, \pi_{\rho}\left(G_{\rho}^{M}\left(Q_{i j}\right)\right) x_{j}\right) \\
& \quad=\sum\left(x_{i}, J_{\rho}^{2} J_{\rho} F_{\rho}^{R^{\prime} R}\left(Q_{i j}\right)^{*} J_{\rho} x_{j}\right) \\
& =\sum\left(J_{\rho}^{2} x_{i}, J_{\rho} F_{\rho}^{R^{\prime} R}\left(Q_{i j}\right)^{*} J_{\rho} x_{j}\right) \\
& =\sum\left(F_{\rho}^{R^{\prime} R}\left(Q_{i j}\right)^{*} J_{\rho} x_{j}, J_{\rho} x_{i}\right) \\
& =\sum\left(J_{\rho} x_{j}, F_{\rho}^{R^{\prime} R}\left(Q_{i j}\right) J_{\rho} x_{i}\right) \geqq 0 .
\end{aligned}
$$


Since $\pi_{\rho}$ is faithful on $s^{M}(\rho) M s^{M}(\rho)$, this proves $n$-positivity of $G_{\rho}^{M}$.

(6) If $\tau^{*} \rho=\rho$, there exists a unitary operator $U_{\rho}(\tau)$ on $H_{\rho}$ such that

$$
U_{\rho}(\tau) \pi_{\rho}(Q) \Omega_{\rho}=\pi_{\rho}(\tau Q) \Omega_{\rho}
$$

Applying $S$, we have

$$
\begin{gathered}
S U_{\rho}(\tau) \pi_{\rho}(Q) \Omega_{\rho}=\pi_{\rho}\left(\tau Q^{*}\right) \Omega_{\rho} \\
=U_{\rho}(\tau) S \pi_{\rho}(Q) \Omega_{\rho} .
\end{gathered}
$$

Hence $U_{\rho}(\tau)$ also commutes with closure $\bar{S}$ and hence with $\Delta_{\rho}$ and $J_{\rho}$. We also have $\tau s^{M}(\rho)=s^{M}(\rho)$. From Theorem 1 (6), we now have, for $\bar{\tau} Q \equiv U_{\rho}(\tau) Q U_{\rho}(\tau)^{*}$,

$$
\begin{aligned}
\pi_{\rho}\left(G_{\rho}^{M}(\tau Q)\right) & =j_{\rho}\left(F_{\rho}^{R^{\prime} R}\left(\bar{\tau} \bar{Q}^{*}\right)\right) \\
& =j_{\rho}\left(\bar{\tau} F_{\rho}^{R^{\prime} R}\left(\bar{Q}^{*}\right)\right) \\
& =\bar{\tau} \pi_{\rho}\left(G_{\rho}^{M}(Q)\right) \\
& =\pi_{\rho}\left(\tau G_{\rho}^{M}(Q)\right) .
\end{aligned}
$$

Since $s^{M}\left(\tau G_{\rho}^{M}(Q)\right) \leqq s^{M}(\tau \rho)=s^{M}(\rho)$, we have $(6)$.

(7) It follows from Theorem 1 (7) and $j_{\pi}\left(\pi_{\rho}\left(s^{M}(z)^{*}\right)\right)=\pi_{\rho}\left(s^{M}(z)\right)$. The latter equation is due to Lemma 5 .

(8) From $G_{\rho}^{M}(Q)=0$ and (3.1), we obtain

$$
\begin{aligned}
0 & =\left(\Omega_{\rho}, \pi_{\rho}(Q) \Delta_{\rho}^{1 / 2} \pi_{\rho}\left(Q^{\prime}\right) \Omega_{\rho}\right) \\
& =\left(\Omega_{\rho}, \pi_{\rho}(Q) j_{\rho}\left(\pi_{\rho}\left(Q^{\prime}\right)^{*}\right) \Omega_{\rho}\right) \\
& =\left(j_{\rho}\left(\pi_{\rho}\left(Q^{\prime}\right)\right) \Omega_{\rho}, \pi_{\rho}(Q) \Omega_{\rho}\right) .
\end{aligned}
$$

Since $j_{\rho}\left(\pi_{\rho}(M)\right) \Omega_{\rho}=\pi_{\rho}(M)^{\prime} \Omega_{\rho}$ span $\pi_{\rho}\left(s^{M}(\rho)\right) H_{\rho}\left(=s^{R}\left(\Omega_{\rho}\right) H_{\rho}\right)$, we have

$$
\pi_{\rho}\left(s^{M}(\rho) Q\right) \Omega_{\rho}=\pi_{\rho}\left(s^{M}(\rho)\right) \pi_{\rho}(Q) \Omega_{\rho}=0 .
$$

By multiplying $Q^{\prime} \in \pi_{\rho}(M)^{\prime}$, we obtain 


$$
\pi_{\rho}\left(s^{M}(\rho) Q s^{M}(\rho)\right) \Psi=\pi_{\rho}\left(s^{M}(\rho) Q\right) s^{M}\left(\Omega_{\rho}\right) \Psi=0
$$

for $s^{R}\left(\Omega_{\rho}\right) \Psi=Q^{\prime} \Omega_{\rho}$ and hence for all $\Psi$. Therefore

$$
\pi_{\rho}\left(s^{M}(\rho) Q s^{M}(\rho)\right)=0
$$

and hence $s^{M}(\rho) Q s^{M}(\rho)=0$. Thus $Q$ must be in $s^{M}(\rho) M\left(1-s^{M}(\rho)\right)+(1-$ $\left.s^{M}(\rho)\right) M$. On the other hand, if $Q$ is in this set, (3.1) vanishes and hence by the uniqueness of $G_{\rho}^{M}(Q)$, we have $G_{\rho}^{M}(Q)=G_{\rho}^{M}(0)=0$.

To prove that the image of $G_{\rho}^{M}$ is strongly dense in $s^{M}(\rho) M s^{M}(\rho)$, it is enough to prove that the image of $G_{\rho}^{M}$ is strongly dense in $M$ for faithful $\rho$ because $\rho$ is faithful on $s^{M}(\rho) M s^{M}(\rho)$. Assume that $\rho$ is faithful on $M$.

Let $\bar{Q} \in \pi_{\rho}(M)$ and

$$
\bar{Q}_{\beta} \equiv \int \tau_{\rho}(t) \bar{Q} \exp \left(-t^{2} / \beta\right) d t /(\beta \pi)^{1 / 2}
$$

It satisfies $\left\|\bar{Q}_{\beta}\right\| \leqq\|Q\|, \lim _{\beta \rightarrow 0} \bar{Q}_{\beta}=\bar{Q} . \quad$ Furthermore,

$$
\tau_{\rho}(t) \bar{Q}_{\beta}=\int \tau_{\rho}(s) \bar{Q} \exp \left(-(t-s)^{2} / \beta\right) d s /(\beta \pi)^{1 / 2}
$$

is analytic for all $t$. Hence, for $Q^{\prime} \in \pi_{\rho}(M)$, we have

$$
\begin{gathered}
\left(\Omega_{\rho},\left(\bar{Q}_{\beta} Q^{\prime}+Q^{\prime} \bar{Q}_{\beta}\right) \Omega_{\rho}\right)=\left(\Omega_{\rho},\left(\bar{Q}_{\beta}+\tau_{\rho}(i) \bar{Q}_{\beta}\right) Q^{\prime} \Omega_{\rho}\right) \\
=\left(\Omega_{\rho},\left(\tau_{\rho}(-i / 2) \bar{Q}_{\beta}+\tau_{\rho}(i / 2) \bar{Q}_{\beta}\right) \Delta_{\rho}^{1 / 2} Q^{\prime} \Omega_{\rho}\right)
\end{gathered}
$$

where the first equality is due to KMS condition. Hence we have for $Q \in M, \bar{Q}=\pi_{\rho}(Q), \bar{Q}_{\beta}=\pi_{\rho}\left(Q_{\beta}\right)$

$$
G_{\rho}^{M}\left(\left\{\tau_{\rho}(-i / 2) Q_{\beta}+\tau_{\rho}(i / 2) Q_{\beta}\right\}\right)=2 Q_{\beta} .
$$

Thus the image of $G_{\rho}^{M}$ is strongly dense in $M$ for faithful $\rho$.

Q.E.D. 


\section{§4. Projections of a von Neumann Algebra into Its Center}

Theorem 3. Let $Z$ denote the center of $M$ and $N \subset Z$. Then $F_{\rho}^{N M}$ has the following properties besides the properties (1)-(8) of Theorem 1.

(9) $F_{\rho}^{N M}$ is a projection from $M$ onto $N s^{N}(\rho)$.

(10) Define $\rho$ and $\rho^{\prime}$ to be $N$-equivalent if $s^{N}(\rho)=s^{N}\left(\rho^{\prime}\right)$ and $\rho^{\prime}$ is in the norm closure of the set of all $A \rho, A \in N, A \geqq 0$. It is an equivalence relation and $F_{\rho}^{N M}=F_{\rho^{\prime}}^{N, M}$ if and only if $\rho$ is $N$-equivalent to $\rho^{\prime}$.

(11) Let $s^{N^{\prime}}\left(\Omega_{\rho}\right)$ be the projection on the closure of $\pi_{\rho}(N) \Omega_{\rho}$. The mapping from $Q \in N s^{N}(\rho)$ to $s^{N^{\prime}}\left(\Omega_{\rho}\right) \pi_{\rho}(Q) \in s^{N^{\prime}}\left(\Omega_{\rho}\right) \pi_{\rho}\left(s^{N}(\rho) M\right)$ is bijective. Let the inverse mapping be $\alpha$. Then

$$
F_{\rho}^{N M}(Q)=\alpha s^{N^{\prime}}\left(\Omega_{\rho}\right) \pi_{\rho}(Q) s^{N^{\prime}}\left(\Omega_{\rho}\right) .
$$

(12) If $K \subset N$, then $F_{\rho}^{K N} F_{\rho}^{N M}=F_{\rho}^{K M}$.

Proof. (9) $F_{\rho}^{N M}(Q)=Q s^{N}(\rho)=Q$ for $Q \in N s(\rho)$ due to Theorem 1 (1) and (2). Hence $F_{\rho}^{N M}$ is a projection onto $N s^{N}(\rho)$.

(10) If $\rho$ is $N$-equivalent to $\rho^{\prime}$, then $\rho^{\prime}$ is a norm limit of $A_{n} \rho$, where we may restrict $s^{N}(\rho) A_{n} \rho \equiv \rho_{n}$. Then by Theorem 1 (7) and (8), we have $F_{\rho^{\prime}}^{N M}(Q)=\lim F_{\rho_{n}}^{N M}(Q)=F_{\rho}^{N M}(Q)$.

Next assume that $F_{\rho}^{N, M}=F_{\rho}^{N M}$. From Theorem 1 (2), we have

$$
s^{N}(\rho)=s^{N}\left(\rho^{\prime}\right)
$$

By the Radon-Nikodym theorem, there exists a non-negative self-adjoint operator $A$ affiliated with $N$ such that $s(A)=s^{N}(\rho)$ and

$$
\rho(Q A)=\rho^{\prime}(Q), \quad Q \in N
$$

Let $E_{\lambda}^{A}$ be the spectral projection of $A$ and $A_{n}=A E_{n}^{A} \in N, \rho_{n}=A_{n} \rho$. Let $\bar{\rho} \equiv A \rho=\lim A_{n} \rho$ which exists as a state of $M$, because $0 \leqq \rho\left(A_{n} Q\right)-$ $\rho\left(A_{m} Q\right) \leqq\|Q\| \rho\left(A_{n}-A_{m}\right) \rightarrow 0$ for $Q \geqq 0, Q \in M$ and $n \geqq m$. Then the restriction of $\bar{\rho}$ to $N$ is the same as the restriction of $\rho^{\prime}$ to $N$. By what we have already proved, $F_{\bar{\rho}}^{N M}=F_{\rho}^{N M}=F_{\rho^{\prime}}^{N M}$. Hence we have 


$$
\bar{\rho}\left(Q Q^{\prime}\right)=\rho^{\prime}\left(Q Q^{\prime}\right)
$$

for all $Q \in M$ and $Q^{\prime} \in N$. Setting $Q^{\prime}=1$, we have $\bar{\rho}=\rho^{\prime}$ as a functional on $M$. This shows that $\rho$ is $N$-equivalent to $\rho^{\prime}$.

$F_{\rho}^{N M}=F_{\rho^{\prime}}^{N, M}$ is certainly an equivalence relation for $\rho$ and $\rho^{\prime}$.

(11) Since $\rho$ is faithful on $N s^{N}(\rho), s^{N^{\prime}}\left(\Omega_{\rho}\right) \pi_{\rho}(Q)=0$ for $Q \in N s^{N}(\rho)$ implies $\left\|s^{N^{\prime}}\left(\Omega_{\rho}\right) \pi_{\rho}(Q) \Omega_{\rho}\right\|^{2}=\rho\left(Q^{*} Q\right)=0$ and hence $Q=0$. Thus $Q \rightarrow s^{N^{\prime}}\left(\Omega_{\rho}\right)$ $\pi_{\rho}(Q)$ is bijective from $N s^{N}(\rho)$ to $s^{N^{\prime}}\left(\Omega_{\rho}\right) \pi_{\rho}\left(N s^{N}(\rho)\right)$.

We have, for $Q \in M, Q^{\prime} \in N$,

$$
\begin{aligned}
\rho\left(Q Q^{\prime}\right) & =\left(\Omega_{\rho}, \pi_{\rho}(Q) \pi_{\rho}\left(Q^{\prime}\right) \Omega_{\rho}\right) \\
& =\left(\Omega_{\rho}, s^{N^{\prime}}\left(\Omega_{\rho}\right) \pi_{\rho}(Q) s^{N^{\prime}}\left(\Omega_{\rho}\right) \pi_{\rho}\left(Q^{\prime}\right) \Omega_{\rho}\right) .
\end{aligned}
$$

If we prove that

$$
s^{N^{\prime}}\left(\Omega_{\rho}\right) \pi_{\rho}(M) s^{N^{\prime}}\left(\Omega_{\rho}\right)=\pi_{\rho}\left(N s^{N}(\rho)\right) s^{N^{\prime}}\left(\Omega_{\rho}\right)
$$

then we have

$$
\left.\rho\left(Q Q^{\prime}\right)=\rho\left(\left\{\alpha s^{N^{\prime}}\left(\Omega_{\rho}\right) \pi_{\rho}(Q) s^{N^{\prime}}\left(\Omega_{\rho}\right)\right)\right\} Q^{\prime}\right) .
$$

Due to the commutativity of elements of $N$, we have (4.1).

To prove (4.2), we note that $\Omega_{\rho}$ is a cyclic vector for abelian $\pi_{\rho}(N)$ on $s^{N^{\prime}}\left(\Omega_{\rho}\right) H_{\rho}$ by definition and hence maximal abelian there. Furthermore, $\pi_{\rho}\left(1-s^{N}(\rho)\right) Q \Omega_{\rho}=0$ for $Q \in \pi_{\rho}(N)$ by the commutativity and hence $s^{N^{\prime}}\left(\Omega_{\rho}\right) \pi_{\rho}\left(s^{N}(\rho)\right)=s^{N^{\prime}}\left(\Omega_{\rho}\right)$. Thus any $Q \in \mathscr{B}\left(s^{N^{\prime}}\left(\Omega_{\rho}\right) H_{\rho}\right)$ satisfying $\left[Q, Q_{1}\right]$ $=0$ for all $Q_{1} \in \pi_{\rho}(N)$ belongs to $\pi_{\rho}\left(N s^{N}(\rho)\right) s^{N^{\prime}}\left(\Omega_{\rho}\right)$.

Since $s^{N^{\prime}}\left(\Omega_{\rho}\right) \in \pi_{\rho}(N)^{\prime}$ and $N$ commutes with $M, Q \in s^{N^{\prime}}\left(\Omega_{\rho}\right) \pi_{\rho}(M)$ $s^{N^{\prime}}\left(\Omega_{\rho}\right)$ commutes with any $Q_{1} \in \pi_{\rho}(N)$. Hence

$$
s^{N^{\prime}}\left(\Omega_{\rho}\right) \pi_{\rho}(M) s^{N^{\prime}}\left(\Omega_{\rho}\right) \subseteq \pi_{\rho}\left(N s^{N}(\rho)\right) s^{N^{\prime}}\left(\Omega_{\rho}\right) .
$$

Since $M \supset N s^{N}(\rho)$, the equality holds.

(12) This is immediate from the defining equations (2.1) and (2.2) and the abelian property of $N$.

Q.E.D.

Corollary. (10), (11) and (12) of Theorem 3 holds if $N \subset M^{\prime}$ and $N$ is abelian. 
Proof. Let $R=(N \cup M)^{\prime \prime}$. Then $N$ is in the center of $R$. Furthermore

$$
F_{\rho}^{N M}(Q)=F_{\rho}^{N R}(Q), \quad Q \in M
$$

Hence by applying Theorem 3 (10) and (11) to $F_{\rho}^{N R}$, we obtain (10) and (11) for $F_{\rho}^{N M}$. Note that $F_{\rho}^{N R}(Q)$ for $Q \in M$ determines $F_{\rho}^{N R}$ due to the property (1) of Theorem 1.

Q.E.D.

Remark. If $N$ is abelian, $Q \in N$ can be identified with continuous function on its spectrum and any normal linear function on $N$ with a Radon measure on its spectrum. Denoting the measure corresponding to the normal linear functional $\rho\left(Q Q^{\prime}\right)=f_{Q}\left(Q^{\prime}\right)$ for $Q^{\prime} \in N$ and $Q \in M$ by $\mu_{Q}$, $F_{\rho}^{N M}$ is given by the Radon-Nikodym derivative:

$$
F_{\rho}^{N M}(Q)=d \mu_{Q} / d \mu_{1}
$$

where we define $d \mu_{Q} / d \mu_{1}=0$ outside the support of $s^{N}(\rho)$.

$F_{\rho}^{N M}(Q)$ for an abelian $N$ has been introduced through the equation (4.1) by D. Ruelle [5] in his theory of decomposition of state. If $\mu_{\rho}$ denotes the measure on the spectrum $\Xi_{N}$ of $N$, corresponding to the restriction of $\rho$ to $N$, then

$$
\rho(Q)=\int_{\Xi_{N}} \xi\left(F_{\rho}^{N M}(Q)\right) d \mu_{\rho}(\xi)
$$

is his decomposition.

\section{§5. Asymptotically Abelian System}

A net $Q_{\alpha}$ of elements of a von Neumann algebra $M$ is called weakly central if there exists a weakly total selfadjoint subset $M_{0}$ of $M$ such that

$$
\left[x, Q_{\alpha}\right] \rightarrow 0
$$

in the weak topology for every $x \in M_{0}$. If (5.1) holds with the strong limit, then $Q_{\alpha}$ is called strongly central. 
The following result is an extension of Proposition 4 of [1] to nonfactors.

Theorem 4. If $Q_{\alpha}$ is a uniformly bounded weakly central net in $M$, then

$$
\mathrm{w}-\lim _{\alpha}\left(Q_{\alpha}-F_{\rho}^{Z M}\left(Q_{\alpha}\right)\right) s^{Z}(\rho)=0
$$

for any normal positive linear functional $\rho$ on $M$, where $Z=M \cap M^{\prime}$.

For any two normal positive linear functionals $\rho$ and $\rho^{\prime}$,

$$
\mathrm{w}-\lim _{\alpha}\left(F_{\rho}^{Z M}\left(Q_{\alpha}\right)-F_{\rho^{\prime}}^{Z, M}\left(Q_{\alpha}\right)\right)\left(s^{Z}(\rho) \wedge s^{Z}\left(\rho^{\prime}\right)\right)=0 .
$$

In particular, if $s^{Z}(\rho)=s^{Z}\left(\rho^{\prime}\right)$,

$$
\mathrm{W}-\lim _{\alpha}\left(F_{\rho}^{Z M}\left(Q_{\alpha}\right)-F_{\rho}^{Z, M}\left(Q_{\alpha}\right)\right)=0 .
$$

When $s^{Z}\left(\rho^{\prime}\right) \leqq s^{Z}(\rho)$, let $A^{Z}\left(\rho^{\prime} / \rho\right)$ be the Radon-Nikodym derivative of $\rho^{\prime}$ by $\rho$ relative to $Z$, namely,

$$
\begin{gathered}
A^{Z}\left(\rho^{\prime} / \rho\right)=\int \lambda d E_{\lambda}, \quad E_{\lambda} \in Z, \\
s\left(A^{Z}\left(\rho^{\prime} / \rho\right)\right)=s^{Z}\left(\rho^{\prime}\right), \\
\rho^{\prime}(z)=\rho\left(z A^{Z}\left(\rho^{\prime} / \rho\right)\right), \quad z \in Z,
\end{gathered}
$$

where $A^{Z}\left(\rho^{\prime} / \rho\right)$ can be unbounded and $\rho\left(z A^{Z}\left(\rho^{\prime} / \rho\right)\right) \equiv \int \lambda d\left(\rho\left(z E_{\lambda}\right)\right)$.

If $s^{Z}\left(\rho^{\prime}\right) \leqq s^{Z}(\rho)$, then

$$
\lim _{\alpha}\left\{\rho^{\prime}\left(Q_{\alpha}\right)-\rho\left(Q_{\alpha} A\left(\rho^{\prime} / \rho\right)\right)\right\}=0 .
$$

In particular, if $A\left(\rho^{\prime} / \rho\right)=1$ (i.e. if $\left.\rho\left|Z=\rho^{\prime}\right| Z\right)$, then

$$
\lim _{\alpha}\left\{\rho^{\prime}\left(Q_{\alpha}\right)-\rho\left(Q_{\alpha}\right)\right\}=0
$$

Proof. Consider $H_{\rho}, \pi_{\rho}, \Omega_{\rho}$ canonically associated with $\rho \neq 0$. Let $\bar{Q}_{\alpha}=\pi_{\rho}\left(Q_{\alpha}\right)$. Let $R_{0}$ be the linear hull of $\pi_{\rho}\left(M_{0}\right), R=\pi_{\rho}(M)=\pi_{\rho}\left(M_{0}\right)^{\prime \prime}=$ $\bar{R}_{0}, \bar{Z}=\pi_{\rho}(Z), s^{\prime}=s^{\bar{Z}^{\prime}}\left(\Omega z_{\rho}\right)$. 
Given $\varepsilon>0$ and vectors $\Phi_{j} \in H, j=1, \ldots, n, \Phi_{j} \neq 0$, there exist $Q_{1}^{\prime \prime}, \ldots$, $Q_{k}^{\prime \prime} \in R_{0}$ and $Q_{1}^{\prime}, \ldots, Q_{k}^{\prime} \in R^{\prime}$ such that

$$
P_{\varepsilon} \equiv \sum_{j=1}^{k} Q_{j}^{\prime \prime} Q_{j}^{\prime}
$$

satisfies

$$
\begin{gathered}
\left\|P_{\varepsilon} \Omega_{\rho}-\Omega_{\rho}\right\|=\left\|\left\{P_{\varepsilon}-s^{\prime}\right\} \Omega_{\rho}\right\| \\
\leqq\left\{\sup _{\alpha}\left\|Q_{\alpha}\right\|\right\}^{-1}\left\{\sup \left\|\Phi_{j}\right\|\right\}^{-1} \varepsilon / 4, \\
\left\|\left\{P_{\varepsilon}^{*}-s^{\prime}\right\} \Phi_{j}\right\| \leqq\left\{\sup _{\alpha}\left\|Q_{\alpha}\right\|\right\}^{-1}\left\|\Omega_{\rho}\right\|^{-1} \varepsilon / 4,
\end{gathered}
$$

because $s^{\prime} \in \bar{Z}^{\prime}$ and linear hull of $R_{0} R_{0}^{\prime}$ is $*$ strongly dense in $\bar{Z}^{\prime}$.

For this set of operators, there exists $\alpha_{\varepsilon}$ such that for all $\alpha>\alpha_{\varepsilon}$,

$$
\left|\left(\Phi_{j},\left[\bar{Q}_{\alpha}, P_{\varepsilon}\right] \Omega_{\rho}\right)\right|<\varepsilon / 2,
$$

due to the weakly central property.

Then for $\alpha>\alpha_{\varepsilon}$, we have

$$
\begin{aligned}
& \left|\left(\Phi_{j},\left[\bar{Q}_{\alpha}, s^{\prime}\right] \Omega_{\rho}\right)\right| \\
& \quad \leqq\left|\left(\Phi_{j},\left[\bar{Q}_{\alpha}, P_{\varepsilon}\right] \Omega_{\rho}\right)\right|+\varepsilon / 2 \\
& \quad<\varepsilon .
\end{aligned}
$$

Hence

$$
\mathrm{W}-\lim _{\alpha}\left[\bar{Q}_{\alpha}, s^{\prime}\right] \Omega_{\rho}=0
$$

By Theorem 3 (11), we have

$$
s^{\prime} \bar{Q}_{\alpha} s^{\prime}=\pi_{\rho}\left(F_{\rho}^{Z M}\left(Q_{\alpha}\right)\right) s^{\prime} .
$$

Since $s^{\prime} \Omega_{\rho}=\Omega_{\rho}$, we obtain from (5.7) and (5.8)

$$
\mathrm{W}-\lim _{\alpha} \pi_{\rho}\left(Q_{\alpha}-F_{\rho}^{Z M}\left(Q_{\alpha}\right)\right) \Omega_{\rho}=0
$$

Take any $Q^{\prime \prime} \in R_{0}, Q^{\prime} \in R_{0}^{\prime}$. Since $\pi_{\rho}\left(F_{\rho}^{Z M}\left(Q_{\alpha}\right)\right) \in \bar{Z}$, it commutes with 
$Q^{\prime \prime} Q^{\prime}$. By weakly central property,

$$
\mathrm{w}-\lim _{\alpha}\left[\pi_{\rho}\left(Q_{\alpha}\right), Q^{\prime \prime} Q^{\prime}\right] \Omega_{\rho}=0
$$

Hence

$$
\mathrm{W}-\lim _{\alpha} \pi_{\rho}\left(Q_{\alpha}-F_{\rho}^{Z M}\left(Q_{\alpha}\right)\right) \Psi=0
$$

for $\Psi=Q^{\prime \prime} Q^{\prime} \Omega_{\rho}$. Since $Q_{\alpha}$ is assumed to uniformly bounded and $\left\|F_{\rho}^{Z M}\right\|=$ 1 , (5.9) holds for all $\Psi$ in the closure of $\bar{Z}^{\prime} \Omega_{\rho}$, which is $s^{\bar{Z}}\left(\Omega_{\rho}\right) H_{\rho}=$ $\pi_{\rho}\left(s^{Z}(\rho)\right) H_{\rho}$. Hence

$$
\mathrm{w}-\lim _{\alpha} \pi_{\rho}\left(\left\{Q_{\alpha}-F_{\rho}^{Z M}\left(Q_{\alpha}\right)\right\} s^{Z}(\rho)\right)=0 .
$$

Since $\pi_{\rho}$ is faithful on $s^{Z}(\rho) M$, we have (5.2).

From (5.2) for $\rho$ and $\rho^{\prime}$, we have (5.3) and in the special case $s^{Z}\left(\rho^{\prime}\right)=s^{Z}(\rho)$, we obtain (5.4), where we use $F_{\rho}^{Z M}\left(Q_{\alpha}\right) s^{Z}(\rho)=F_{\rho}^{Z M}\left(Q_{\alpha}\right)$.

If $s^{Z}\left(\rho^{\prime}\right) \leqq s^{Z}(\rho)$, we obtain from (5.2)

$$
\lim _{\alpha}\left\{\rho^{\prime}\left(Q_{\alpha}\right)-\rho^{\prime}\left(F_{\rho}^{Z M}\left(Q_{\alpha}\right)\right)\right\}=0 .
$$

Using the definition of $A^{Z}\left(\rho^{\prime} / \rho\right)$ and (2.1) with $Q^{\prime}=1$, we obtain

$$
\begin{aligned}
& \rho^{\prime}\left(F_{\rho}^{Z M}\left(Q_{\alpha}\right)\right)=\int \lambda d \rho\left(F_{\rho}^{Z M}\left(Q_{\alpha}\right) E_{\lambda}\right) \\
& =\int \lambda d \rho\left(Q_{\alpha} E_{\lambda}\right)=\rho\left(Q_{\alpha} A^{Z}\left(\rho^{\prime} / \rho\right)\right) .
\end{aligned}
$$

This proves (5.5). (5.6) then follows.

Q.E.D.

If a subset 2 . of a von Neumann algebra $M$ and a net of $*$ automorphisms $\tau_{\alpha}$ of $M$ satisfy the property that $\tau_{\alpha} Q$ for every $Q \in \mathfrak{A}$ is weakly (or strongly) central, then $\mathfrak{Q}$ is called weakly (or strongly) $\tau_{\alpha}$ central in $M$.

Corollary. If $\mathfrak{U}$ is weakly $\tau_{\alpha}$ central in $M$ and $\rho$ is $a \tau_{\alpha}$ invariant normal positive linear functional on $M$, then

$$
\mathrm{w}-\lim _{\alpha}\left(\tau_{\alpha} Q-\tau_{\alpha} F_{\rho}^{Z M}(Q)\right) s^{Z}(\rho)=0
$$


for all $Q \in \mathfrak{N}$ where $Z$ is the center of $M$.

If $\rho^{\prime}$ is another normal positive linear functional on $M$ and $s^{Z}\left(\rho^{\prime}\right) \leqq$ $s^{Z}(\rho)$, then

$$
\lim _{\alpha}\left\{\rho^{\prime}\left(\tau_{\alpha} Q\right)-\rho\left(Q \tau_{\alpha}^{-1} A^{Z}\left(\rho^{\prime} / \rho\right)\right)\right\}=0
$$

for all $Q \in \mathfrak{U}$ where $\tau_{\alpha}^{-1} A^{Z}\left(\rho^{\prime} / \rho\right)=\int \lambda d\left(\tau_{\alpha}^{-1} E_{\lambda}\right)$. In particular if $\rho^{\prime}(z)=$ $\rho(z)$ for all $z \in Z$, then

$$
\lim _{\alpha} \rho^{\prime}\left(\tau_{\alpha} Q\right)=\rho(Q), \quad Q \in \mathfrak{R}
$$

Proof. Since $\left\|\tau_{\alpha} Q\right\|=\|Q\|, \tau_{\alpha} Q$ is uniformly bounded. By (5.2), (2.3) and $\tau_{\alpha}^{*} \rho=\rho$, we have (5.11). (5.12) follows from (5.5) and the invariance of $\rho .(5.13)$ is a special case of (5.12) where $s^{Z}\left(\rho^{\prime}\right)=s^{Z}(\rho)$ and $A^{Z}\left(\rho^{\prime} / \rho\right)$ $=1$.

Q.E.D.

Remark. If $Q_{\alpha}$ is weakly central and uniformly bounded, then w-lim $\left[x, Q_{\alpha}\right]=0$ for all $x \in M$, because it holds for any $x$ in the linear hull $M_{1}$ of $M_{0}$, which, being a weakly dense linear subset, is $*$ strongly dense in $M$, and hence for given $x \in M, \varepsilon>0, \Psi_{j}, \Phi_{j}$, there exist $x^{\prime} \in M_{1}$ and $\alpha_{0}$ such that $\left\|Q_{\alpha}\right\|<L$,

$$
\left\|\Psi_{j}\right\| L\left\|\left(x-x^{\prime}\right) \Phi_{j}\right\|<\varepsilon / 3,\left\|\left(x-x^{\prime}\right) * \Psi_{j}\right\| L\left\|\Phi_{j}\right\|<\varepsilon / 3
$$

and

$$
\left|\left(\Psi_{j},\left[x^{\prime}, Q_{\alpha}\right] \Phi_{j}\right)\right|<\varepsilon / 3 \quad \text { for } \alpha>\alpha_{0}
$$

which imply $\left|\left(\Psi_{j},\left[x, Q_{\alpha}\right] \Phi_{j}\right)\right|<\varepsilon, j=1, \ldots, n$.

Hence, if $\mathfrak{Q}$ is weakly $\tau_{\alpha}$ central, then the norm closure $\mathfrak{A}_{1}$ of the linear hull of $\mathfrak{A} \cup \mathfrak{A}^{*}$ is obviously weakly $\tau_{\alpha}$ central and (5.2)-(5.6) for $Q_{\alpha}=\tau_{\alpha} Q$ and (5.11)-(5.13) hold for any $Q \in \mathfrak{U}_{1}$.

(5.11)-(5.13) hold for $\sigma$-weak colsure of $\mathfrak{U}_{1}$ if $s^{Z}(\rho)$ and $s^{Z}\left(\rho^{\prime}\right) \leqq s^{Z}(\rho)$ are replaced by $s^{M}(\rho)$ and $s^{M}\left(\rho^{\prime}\right) \leqq s^{M}(\rho)$, because (5.11) implies

$$
\mathrm{w}-\lim \pi_{\rho}\left(\tau_{\alpha} Q-\tau_{\alpha} F_{\rho}^{Z M}(Q)\right) \Psi=0
$$


for $\Psi=\Omega_{\rho}$ and $Q$ in the strong closure of the unit ball of $\mathfrak{U}_{1}$ and hence for $\Psi \in \overline{R^{\prime} \Omega_{\rho}}$ and $Q$ in the $\sigma$-weak closure of $\mathfrak{U}_{1}$.

The next theorem has an application in [2].

Description of situation. A von Neumann algebra $M$, a net of * automorphisms $\tau_{\alpha}$, a faithful normal positive linear functional $\rho \neq 0$ on $M$, invariant under all $\tau_{\alpha}$ and a $C^{*}$ subalgebra $\mathfrak{Q}$ of $M$ are given. Let $U_{\alpha}$ be the unique unitary operator on $H_{\rho}$ satisfying $U_{\alpha} \pi_{\rho}(Q) \Omega_{\rho}=\pi_{\rho}$ $\left(\tau_{\alpha} Q\right) \Omega_{\rho}$ for all $Q \in M$. Let $\bar{\tau}_{\alpha} Q \equiv U_{\alpha} Q U_{\alpha}^{*}$ for all $Q \in \mathscr{B}\left(H_{\rho}\right)$. Let $J_{\rho}$ be the modular conjugation operator for the cyclic and separating $\Omega_{\rho}$ relative to $\pi_{\rho}(M)$ and $j_{\rho}(Q) \equiv J_{\rho} Q J_{\rho}, Q \in \mathscr{B}\left(H_{\rho}\right)$. Let $\hat{\mathfrak{U}}$ be the $C^{*}$ algebra generated by

$$
\pi_{\rho}(\mathfrak{U}) j_{\rho}\left\{\pi_{\rho}(\mathfrak{Z})\right\}
$$

and $\hat{R} \equiv\left(\pi_{\rho}(M) \cup \pi_{\rho}(M)^{\prime}\right)^{\prime \prime}$.

Theorem 5. Assume that $\mathfrak{2}$ is strongly $\tau_{\alpha}$ central in $M$. For any normal positive linear functional $\rho^{\prime}$ on $\mathscr{B}\left(H_{\rho}\right)$, all $Q \in \hat{\mathfrak{Q}}$ satisfy

$$
\lim _{\alpha}\left\{\rho^{\prime}\left(\bar{\tau}_{\alpha} Q\right)-\left(\Omega_{\rho}, Q \bar{\tau}_{\alpha}^{-1} A^{\bar{Z}}\left(\rho^{\prime} / \bar{\rho}\right) \Omega_{\rho}\right)\right\}=0
$$

where $\bar{\rho}=\omega_{\Omega}, \bar{Z}=\pi_{\rho}(M) \cap \pi_{\rho}(M)^{\prime}$ which is the center of $\hat{R}$ and $A^{\bar{Z}}\left(\rho^{\prime} / \bar{\rho}\right)$ is as in Theorem 4. In particular, if $\rho(z)=\rho^{\prime}\left(\pi_{\rho}(z)\right)$ for all $z$ in the center of $M$, then

$$
\lim _{\alpha} \rho^{\prime}\left(\bar{\tau}_{\alpha} Q\right)=\bar{\rho}(Q), \quad Q \in \hat{\mathfrak{A}} \text {. }
$$

Proof. Let $S_{\rho}=J_{\rho} \Delta_{\rho}^{1 / 2}$. We have

$$
\begin{aligned}
U_{\alpha} S_{\rho} Q \Omega_{\rho} & =U_{\alpha} Q^{*} \Omega_{\rho}=\left(\bar{\tau}_{\alpha} Q\right)^{*} \Omega_{\rho} \\
& =S_{\rho}\left(\bar{\tau}_{\alpha} Q\right) \Omega_{\rho}=S_{\rho} U_{\alpha} Q \Omega_{\rho}
\end{aligned}
$$

where $Q \in \pi_{\rho}(M)$, which implies $\bar{\tau}_{\alpha} Q \in \pi_{\rho}(M)$. Thus $U_{\alpha}$ commutes with $S_{\rho}$ and hence with $\Delta_{\rho}=S_{\rho}^{*} S_{\rho}$ and $J_{\rho}$.

Let $Q, Q^{\prime} \in \mathfrak{N}, Q_{0} \in M_{0}(Q), Q_{0}^{\prime} \in M_{0}\left(Q^{\prime}\right)$ where $M_{0}(Q)$ is a selfadjoint 
total subset of $M$ such that (5.1) is satisfied in the strong topology for all $x \in M_{0}(Q)$ and $Q_{\alpha}=\tau_{\alpha}(Q)$ and $M_{0}\left(Q^{\prime}\right)$ is the same for $Q^{\prime}$.

$$
\begin{aligned}
& {\left[\pi_{\rho}\left(Q_{0}\right) j_{\rho}\left\{\pi_{\rho}\left(Q_{0}^{\prime}\right)\right\}, \bar{\tau}_{\alpha}\left(\pi_{\rho}(Q) j_{\rho}\left\{\pi_{\rho}\left(Q^{\prime}\right)\right\}\right)\right]} \\
& =\pi_{\rho}\left(\left[Q_{0}, \tau_{\alpha} Q\right]\right) j_{\rho}\left\{\pi_{\rho}\left(Q_{0}^{\prime} \tau_{\alpha} Q^{\prime}\right)\right\} \\
& \quad+\pi_{\rho}\left(\left\{\tau_{\alpha} Q\right\} Q_{0}\right) j_{\rho}\left\{\pi_{\rho}\left(\left[Q_{0}^{\prime}, \tau_{\alpha} Q^{\prime}\right]\right)\right\} .
\end{aligned}
$$

Since both $\left[Q_{0}, \tau_{\alpha} Q\right]$ and $\left[Q_{0}^{\prime}, \tau_{\alpha} Q^{\prime}\right]$ tends to 0 strongly, and all operators are bounded uniformly in $\alpha$, (5.17) tends to 0 strongly.

Since $M_{0}(Q)$ and $M_{0}\left(Q^{\prime}\right)$ are selfadjoint and total,

$$
\hat{M}_{0}=\pi_{\rho}\left(M_{0}(Q)\right) j_{\rho}\left\{\pi_{\rho}\left(M_{0}\left(Q^{\prime}\right)\right)\right\}
$$

is also selfadjoint and total in $\hat{R}$. Hence (5.14) is strongly $\tau_{\alpha}$ central in $\hat{R}$.

By (5.12) and (5.13), we obtain (5.15) and (5.16) when $Q$ is in (5.14). Note that $s^{Z}(\rho)=1$ because $\rho$ is assumed to be faithful. Note also that $\bar{Z}=\pi_{\rho}(Z)$.

By Remark after Corollary to Theorem 4, $Q$ in (5.15) and (5.16) can be in the norm closure of the linear hull of (5.14), which is $\hat{\mathfrak{U}}$.

Q.E.D.

Remark. Let $\mathfrak{U}_{1}$ be a $C^{*}$ algebra, $\rho$ be a state on $\mathfrak{U}_{1}$ and $\tau_{\alpha}$ be a net of $*$ automorphisms of $\mathfrak{U}_{1}$. If

$$
\pi_{\rho}\left(\left[Q_{1}, \tau_{\alpha} Q_{2}\right]\right)
$$

tends to 0 weakly (or strongly) for all $Q_{1}, Q_{2} \in \mathfrak{A}_{1}$, then $\mathfrak{A}_{1}$ is said to be weakly (or strongly) $\tau_{\alpha}$ asymptotically abelian. We can apply Theorem 4 to such a situation by taking $M_{0}=\pi_{\rho}\left(\mathfrak{U}_{1}\right), M=\pi_{\rho}\left(\mathfrak{U}_{1}\right)^{\prime \prime}$ and $Q_{\alpha}=\pi_{\rho}\left(\tau_{\alpha} Q\right)$ for $Q \in \mathfrak{A}_{1}$. If $\rho$ is $\tau_{\alpha}$ invariant and $\mathfrak{A}_{1}$ is strongly $\tau_{\alpha}$ asymptotically abelian, then we can apply Theorem 5 .

Method of big translation in $[3]$ can be formulated as follows. (See Theorem 6). 
Lemma 6. Let $\rho_{\alpha}$ be a net of (not necessarily normal) positive linear functionals on $(M \cup N)^{\prime \prime}$ such that $\lim _{\alpha} \rho_{\alpha}=\rho$ (i.e. $\lim _{\alpha} \rho_{\alpha}(Q)=\rho(Q)$ for each $\left.Q \in(M \cup N)^{\prime \prime}\right)$. Assume that the restriction of $\rho_{\alpha}$ to $N$ is normal and independent of $\alpha$. Assume also that $N$ is abelian. Then

$$
\mathrm{w}-\lim _{\alpha} F_{\rho_{\alpha}}^{N M}(Q)=F_{\rho}^{N M}(Q), \quad Q \in M
$$

(See Remark 2 of §2.)

Proof. Let the restriction of $\rho_{\alpha}$ to $N$ be denoted by $\sigma$ which is independent of $\alpha$ by assumption. Then we obtain, from $\lim _{\alpha} \rho_{\alpha}=\rho$,

$$
\lim _{\alpha} \sigma\left(F_{\rho_{\alpha}}^{N M}(Q) Q_{1}^{\prime} Q_{2}^{\prime}\right)=\sigma\left(F_{\rho}^{N M}(Q) Q_{1}^{\prime} Q_{2}^{\prime}\right)
$$

for all $Q_{1}^{\prime} \in N, Q_{2}^{\prime} \in N$. Setting $x_{\alpha}=\left(F_{\rho_{\alpha}}^{N M}-F_{\rho}^{N M}\right)(Q)$, we have

$$
\lim _{\alpha}\left(\Psi, \pi_{\sigma}\left(x_{\alpha}\right) \Phi\right)=0
$$

for $\Psi=\pi_{\sigma}\left(Q_{1}^{\prime}\right)^{*} \Omega_{\sigma}$ and $\Phi=\pi_{\sigma}\left(Q_{2}^{\prime}\right) \Omega_{\sigma}$. Since $\left\|x_{\alpha}\right\| \leqq\left. 2\right|_{\mid} ^{\prime} Q \|$, we have w-lim $\pi_{\sigma}\left(x_{\alpha}\right)=0$. Since $s\left(x_{\alpha}\right) \leqq s^{N}(\sigma)$, we obtain $\mathrm{w}-\lim _{\alpha} x_{\alpha}=0$.

Theorem 6. Let $\mathfrak{A}$ be a weakly $\tau_{\alpha}$ central $C^{*}$ subalgebra of a von Neumann algebra $M$. Assume that the center $Z$ of $M$ is elementwise $\tau_{\alpha}$ invariant and has a faithful normal state $\rho$. Let $\overline{\mathfrak{A}}$ be the $C^{*}$ algebra generated by $\mathfrak{X}$ and $Z$. Then there exists a subnet $\tau_{\alpha(\beta)}$ such that

$$
L(Q)=\mathrm{w}-\lim _{\beta} \tau_{\alpha(\beta)} Q
$$

exists for all $Q \in \overline{\mathfrak{N}}$, where $L$ is Z-linear, completely positive projection of norm 1 from $\overline{\mathfrak{N}}$ onto $Z$ and $L(1)=1$. If $Z$ is trivial, then $L(Q)=\omega(Q) 1$ for a state $\omega$ on $\overline{\mathfrak{U}}$.

Proof. Let $\bar{\rho}$ be any extension of $\rho$ to a state on $M$. By weak compactness, there exists a subnet $a(\beta)$ such that

$$
\lim \tau_{\alpha(\beta)}^{*} \bar{\rho}=\rho_{\infty}
$$

exists. 
Since $Z$ is elementwise invariant under $\tau_{\alpha}$, the restriction of $\tau_{\alpha}^{*} \bar{\rho}$ to $Z$ is always $\rho$ and hence the restriction of $\rho_{\infty}$ to $Z$ is also $\rho$. Since $\rho$ is faithful on $Z, s^{Z}(\rho)=1$. By (5.2), (2.3) and Lemma 6,

$$
\begin{aligned}
\mathrm{w}-\lim _{\beta} \tau_{\alpha(\beta)} Q=\mathrm{w}-\lim _{\beta} F_{\bar{\rho}}^{Z M}\left(\tau_{\alpha(\beta)} Q\right) \\
=\mathrm{w}-\lim _{\beta} F_{\tau_{\alpha(\beta)}^{*}}^{Z M}(Q) \\
=F_{\rho_{\infty}}^{Z M}(Q) .
\end{aligned}
$$

Hence (5.19) holds with $L=F_{\rho_{\infty}}^{Z M}$. The properties of $L$ follow from Theorem 1 applied for $F_{\rho_{\infty}}^{Z M}$ (see Remark 2 of $\S 2$ ) except possibly for the complete positivity.

Since $Z$ is abelian, $J_{\rho} z^{*} J_{\rho}=z, z \in \pi_{\rho}(Z)$ for a faithful state $\rho$. Hence $Q \rightarrow^{t} Q \equiv J_{\rho} Q^{*} J_{\rho}$ is a transposition on $\mathscr{B}\left(H_{\rho}\right)$ leaving $Z$ invariant. Hence if $L$ is transposed- $n$-positive then

$$
L \otimes 1_{n}=\left(\pi_{\rho}^{-1} \otimes 1_{n}\right)\left(t \otimes t_{n}\right)\left(\pi_{\rho} \otimes 1_{n}\right)\left(L \otimes t_{n}\right)
$$

is also positive and hence $F$ is $n$ positive. Here $1_{n}$ and $t_{n}$ denote the identity mapping and a transposition of $n \times n$ matrices. $\quad$ Q.E.D.

\section{Acknowledgement}

This work has been carried out at Department of Mathematics, Queen's University. The author would like to thank the members of the Department of Mathematics, Queen's University for their warm hospitality and discussions.

\section{References}

[1] Araki, H., Progr. Theoret. Phys. 32 (1964), 844-854.

[2] Araki, H., Comm. Math. Phys. 28 (1972), 267-277.

[3] Borchers, H. J., Comm. Math. Phys. 1 (1965), 57-79.

[4] Doplicher, S., R. V. Kadison, D. Kastler \& D. W. Robinson, Comm. Math. Phys. 6 (1967), 101-120.

[5] Ruelle, D., J. Functional Analysis, 6 (1970), 116-151.

[6] Sakai, S., $C^{*}$ Algebras and $W^{*}$ Algebras, Springer-Verlag, New York-HeidelbergBerlin, 1971. 
[7] Stinespring. W. F., Proc. Amer. Math. Soc. 6 (1955), 211-216.

[8] Takesaki, M., Tomita's Theory of Modular Hilbert Algebras and Its Applications, Springer Verlag, Berlin-Heidelberg-New York, 1970.

[9] Umegaki, H., Kōdai Math. Sem. Rep. 11 (1959), 51-64.

[10] Nakamura, M., M. Takesaki and H. Umegaki, Kōdai Math. Sem. Rep. 12 (1960), 82-90. 
